\title{
A bounded upwinding scheme for computing convection-dominated transport problems
}

\author{
V.G. Ferreira ${ }^{\text {a,* }}$, R.A.B. de Queiroz $^{\text {a }}$, G.A.B. Lima ${ }^{a}$, R.G. Cuenca ${ }^{a}$, C.M. Oishi ${ }^{b}$, J.L.F. Azevedo ${ }^{c}$, S. McKee $^{\mathrm{d}}$ \\ a Departamento de Matemática Aplicada e Estatística, Instituto de Ciências Matemáticas e de Computação - USP, São Carlos, SP, Brazil \\ ${ }^{\mathrm{b}}$ Departamento de Matemática, Universidade Estadual Júlio de Mesquita Filho - UNESP, Presidente Prudente, SP, Brazil \\ ${ }^{\mathrm{c}}$ Instituto de Aeronautica e Espaço, CTA/IAE/ALA, São José dos Campos, SP, Brazil \\ ${ }^{\mathrm{d}}$ Department of Mathematics and Statistics, University of Strathclyde, Glasgow, UK
}

\section{A R T I C L E I N F O}

\section{Article history:}

Received 20 April 2009

Received in revised form 26 September

2011

Accepted 27 December 2011

Available online 10 January 2012

\section{Keywords:}

Numerical simulation

CBC/TVD stability

High resolution

Upwinding

Monotonic interpolation

Finite difference

Convection modeling

Boundedness

\begin{abstract}
A B S T R A C T
A practical high resolution upwind differencing scheme for the numerical solution of convection-dominated transport problems is presented. The scheme is based on TVD and CBC stability criteria and is implemented in the context of the finite difference methodology. The performance of the scheme is investigated by solving the 1D/2D scalar advection equations, 1D inviscid Burgers' equation, 1D scalar convection-diffusion equation, 1D/2D compressible Euler's equations, and 2D incompressible NavierStokes equations. The numerical results displayed good agreement with other existing numerical and experimental data.
\end{abstract}

(c) 2012 Elsevier Ltd. All rights reserved.

\section{Introduction}

The development of fast, reliable and accurate numerical approximations for the convection terms of hyperbolic conservation laws and transport equations in fluid dynamics has presented a continuing challenge. The most frustrating obstacle has been the attempt to prevent the unbounded growth of the unphysical spatial oscillations in the vicinity of sharp changes in gradients, or jump discontinuities. It is also essential that certain transport variables remain bounded within physical limiting values. For example, the fluid depth in shallow water flows, the mixture fraction of reacting flows, the kinetic energy in turbulent flows, or species concentration all cannot fall below zero. Previous studies by Smith and Hutton [54] (see also van Albada et al. [64] and van Leer [69]) have shown that upwinding schemes may produce nonphysical results when boundedness is not preserved.

In order to obtain stable, bounded and physically plausible solutions, the classical first order upwind (FOU) difference scheme [21] - or the hybrid central upwind (HCU) $[48,58]$ - is often adopted.

* Corresponding author. Address: Av. Trabalhador São-carlense, 400 - Centro, CEP: 13560-970, São Carlos, SP, Brazil.

E-mail address: pvgf@icmc.usp.br (V.G. Ferreira).
However, this scheme is generally unsuitable for applications involving long time evolution of complex flows (unless extremely fine meshes are employed), mainly because extrema can become "clipped" and numerical dissipation (even spatial derivatives) can become dominant (see Refs. [13,46,59]).

The cure for this has been to use conventional schemes, such as central differences (CDs), second-order upwind (SOU) [70], and quadratic-upstream interpolation for convective kinematics (QUICK) [39] (or its related QUICK with estimated streamline terms (QUICKEST) [41]), to name just a few. However, under highly convective conditions, these schemes also inevitably generate spurious numerical (or non-monotonic) oscillations (wild [17] or parasitic solutions [25]) and instabilities in regions where the convected variables experience discontinuities.

To overcome these defects, a number of monotonic high-order upwind schemes have appeared in the published literature such as, for example, the sharp and monotonic algorithm for realistic transport (SMART) [24], the simple high accuracy resolution program (SHARP) [40], the variable-order non-oscillatory scheme (VONOS) [65], the weighted-average coefficient ensuring boundedness (WACEB) [57], the convergent and universally bounded interpolation scheme for the treatment of advection (CUBISTA) [5], and an adaptive bounded version of the QUICKEST (ADBQUICKEST) [23]. 
In addition, from a more "compressible" point of view, one may add to this list the monotone upstream scheme for conservation laws (MUSCL) originally pioneered by van Leer [68] and the associated limiters developed for the past 20 years: van Leer [66,67], van Albada [64] (and its variants), Koren [35], Osher [47], Superbee [7], Minmod [28], among others. The main objective of these schemes is to recover smooth solutions from those that are contaminated by oscillations and, at the same time, to improve the rate of convergence. It should be also noted, however, that these schemes (some of them at least), though performing well on some problems, cannot be bounded in situations such as shock phenomena in compressible flows (see, for instance [37,44]) and/or incompressible viscoelastic flow calculations with hyperbolic constitutive models (see, for instance [73]). Lin and Chieng [43] and Lin and Lin [44], for example, observed that the SMART and SHARP schemes, although preserving high-order accuracy, produce high levels of oscillations in the case of the unsteady one-dimensional shock tube problem; Alves et al. [4], using high-order upwind schemes, ran a series of tests to simulate viscoelastic flows and observed that the computations suffered from convergence difficulties when the mesh was refined, and had a strong tendency to oscillate.

Hence, the need for simple, accurate, efficient and robust upwind differencing schemes for approximating nonlinear convective terms of conservation laws and related unsteady fluid dynamics equations continues to stimulate a great deal of research. This is the prime motivation for the upwind scheme presented in this work. Further motivation for development of upwind differencing schemes for approximating convective terms lies in the desire of the authors to develop a numerical technique that will be equally applicable both to compressible and incompressible problems.

Possibly because advection is one of the most expensive processes in many numerical models, it is not surprising that mathematically equivalent high resolution upwind schemes have been invented independently, often from a different conceptual basis. For example, the SMARTER scheme of Choi and his co-authors [19] (see also the original Ref. [55]) is equivalent to the ISNAS of Zijlema [76] and the CHARM of Zhou et al. [77]; the CROWLEY scheme of Tremback et al. [63] is equivalent to the QUICKEST of Leonard [41]; and HARMONIC of van Leer [68] was renamed as HLPA by Zhu [79].

In this work, a new high resolution upwind scheme, called TOPUS (Third-Order Polynomial Upwind Scheme) is presented for simulating compressible and incompressible flows; it may be viewed as a generalization of the SMARTER scheme (see [19]) and follows the basic idea of constructing a numerical flux function using a combination of low and high order schemes through some switching function (limiter), which assesses local variation in the solution. This scheme approximates the advective fluxes at the cell boundaries with $1 \mathrm{st}$, 2 nd or 3rd order accuracy and displays little dissipation at high wave number. The expectation is that the use of this new polynomial upwind scheme will enable us not merely to capture a shock, but also to resolve the delicate features and structures of complex flows. In the derivation of the TOPUS scheme, the total variation diminishing (TVD) and convection boundedness criterion $(\mathrm{CBC})$ are employed for the stability of the solution; they also offer some flexibility in the construction of the higher-order upwind bounded schemes.

It is important to bear in mind that there exists another very successful class of high resolution shock-capturing schemes, namely, the essentially non-oscillatory (ENO) [34] (and its related weighted ENO (WENO) [9]). However, in comparison with the TOPUS scheme, the implementation of the ENO scheme can be difficult. For example, when dealing with systems of equations, the ENO scheme requires the decomposition of the characteristic variables applied to each component of the vector of the characteristic variable; then the numerical flux is required to be transformed back to physical space (for more details, see [74]). Nonetheless, there is no doubt that the ENO and WENO schemes are excellent methods for compressible flow computation.

The main focus of the paper is to put forward an alternative universal numerical technique which can cope with both compressible and incompressible fluid flows. However, the paper may also be regarded as a review of existing bounded upwinding schemes, the best of which (in the authors' opinion) have been implemented so that a comparison may be made with TOPUS.

The structure of the paper is as follows. In Section 2, we present the mathematical formulation of the TOPUS scheme, a discussion about the implementation of the scheme and finally a summary of those schemes that are to be compared with TOPUS. The numerical solutions for 1D and 2D problems are presented in Section 3 to illustrate the versatility and robustness of TOPUS. Section 4 contains a few concluding remarks.

\section{The TOPUS scheme and its implementation}

In this section, the TOPUS scheme will be derived and then issues concerning implementation will be discussed. Also, a listing of the schemes to be compared with TOPUS will be presented.

\subsection{Description of the scheme}

Before proceeding to the derivation of the TOPUS convective scheme, it is essential to introduce the normalized variables (NV) of Leonard [41] and the conditions required for the construction of a monotonic upwinding scheme [40,41] (using the CBC criterion of Gaskell and Lau [24]). To clarify our approach, consider the 1D linear advection equation

$\frac{\partial \phi}{\partial t}+a \frac{\partial \phi}{\partial x}=0$

together with appropriate initial and boundary conditions. In Eq. (1), $\phi=\phi(x, t)$ is the dependent variable and $a$ is the convection speed (constant). The solution of this equation can be approximated by the conservative finite difference method

$\phi_{i}^{n+1}=\phi_{i}^{n}-\theta\left(\phi_{i+1 / 2}^{n}-\phi_{i-1 / 2}^{n}\right)$,

where $\phi_{i}^{n}$ is the numerical solution at mesh point $\left(i \delta_{x}, n \delta_{t}\right)$, with $\delta_{x}$ and $\delta_{t}$ being space and time increments in the $x$-and $t$-directions, respectively, and $\theta=a \frac{\delta_{t}}{\delta_{x}}$ is the Courant number. In the above equation, $\phi_{i+1 / 2}^{n}$ and $\phi_{i-1 / 2}^{n}$, denoted by $\phi_{f}$ and $\phi_{g}$ respectively (see Fig. 1 ), are approximations for the convected variable $\phi$ which, in this paper, will be calculated, according to the sign of the local advection velocity, $V_{(\cdot)}=V_{\text {(through a control surface) }}$, as a function of the values at three selected neighboring points (two upwind, $U$ and $R$, and one downwind $D$ ). For example, in Fig. 1 the $f$ face is presented together with its advection velocity $V_{f}>0$ and neighboring nodes $i+1=D$, $i=U$ and $i-1=R$. The variation of a convected quantity $\phi$ through, for example, the boundary face $f$ between two control volumes can

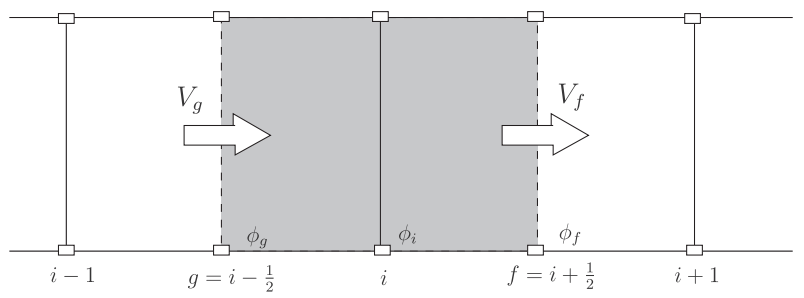

Fig. 1. Advection velocities through $f$ and $g$ faces, and neighboring nodes of these faces. 
be represented by a functional relationship linking values $\phi_{D}, \phi_{U}$ and $\phi_{R}$, which represent, respectively, the Downstream, the Upstream and the Remote-upstream locations with respect to the advection velocity $V_{f}$ at this face. The neighbors of the $g$ face can be similarly classified. If this functional relationship, involving these three neighboring positions, is prescribed, then the value of the interface convected variable can be determined. To this end, the original variable $\phi$ is transformed into the NV of Leonard [41] by

$\hat{\phi}(x, t)=\frac{\phi(x, t)-\phi_{R}^{n}}{\phi_{D}^{n}-\phi_{R}^{n}}$.

The advantage of this normalization is that the interface value $\hat{\phi}_{f}$ depends on $\hat{\phi}_{U}^{n}$ and $\theta$ only, since $\hat{\phi}_{D}^{n}=1$ and $\hat{\phi}_{R}^{n}=0$. From now on, the superscript $n$ will be omitted for simplicity. The CBC is a condition for achieving computed boundedness if only three neighboring values are used to approximate the interface numerical convected variables. According to Leonard [40,41], a bounded high resolution second and/or third order accurate scheme (in general, nonlinear) within the $C B C$ region must pass through points $O(0,0)$, $Q(0.5,0.75), P(1,1)$ and with inclination of 0.75 at $Q$. Passing through $Q$ will provide second order accuracy and passing through $Q$ with a slope of 0.75 will give third order accuracy.

The TOPUS scheme is derived by assuming that the NV at the cell interface $f, \hat{\phi}_{f}$, are related to $\hat{\phi}_{U}$ by a fourth degree polynomial function for $0<\hat{\phi}_{U}<1$, and a linear function (the FOU scheme) for $\hat{\phi}_{U} \leqslant 0$ and $\hat{\phi}_{U} \geqslant 1$. The four conditions of Leonard presented above, plus a free condition, are imposed to obtain

$\hat{\phi}_{f}=\left\{\begin{array}{l}\alpha \hat{\phi}_{U}^{4}+(-2 \alpha+1) \hat{\phi}_{U}^{3}+\left(\frac{5 \alpha-10}{4}\right) \hat{\phi}_{U}^{2}+\left(\frac{-\alpha+10}{4}\right) \hat{\phi}_{U}, \hat{\phi}_{U} \in(0,1), \\ \hat{\phi}_{U}, \quad \hat{\phi}_{U} \notin(0,1),\end{array}\right.$

where $\alpha$ is an adjustable constant in the interval [ $-2,2]$. If $\alpha=0$, then TOPUS falls into the CBC region of Gaskell and Lau [24] and corresponds to the SMARTER scheme of Choi and co-authors [19] (see also Waterson and Deconinck [71]). By imposing an inclination of 1 at point $P$ (i.e. a continuously differentiable function at $P$ ), one obtains $\alpha=2$. Fig. 2 depicts the TOPUS scheme for the case $\alpha=2$, where one can see that it is entirely contained within the TVD region of Harten [29]. Other values of $\alpha$ ensure that TOPUS falls within the $\mathrm{CBC}$ region. In practice, it is necessary to be careful. For incompressible flows, it can be chosen from $[-2,2]$ and boundedness will be ensured; a good choice is $\alpha=0$ or $\alpha=2$. For compressible flows, one must set $\alpha=2$ to guarantee the TVD criterion.

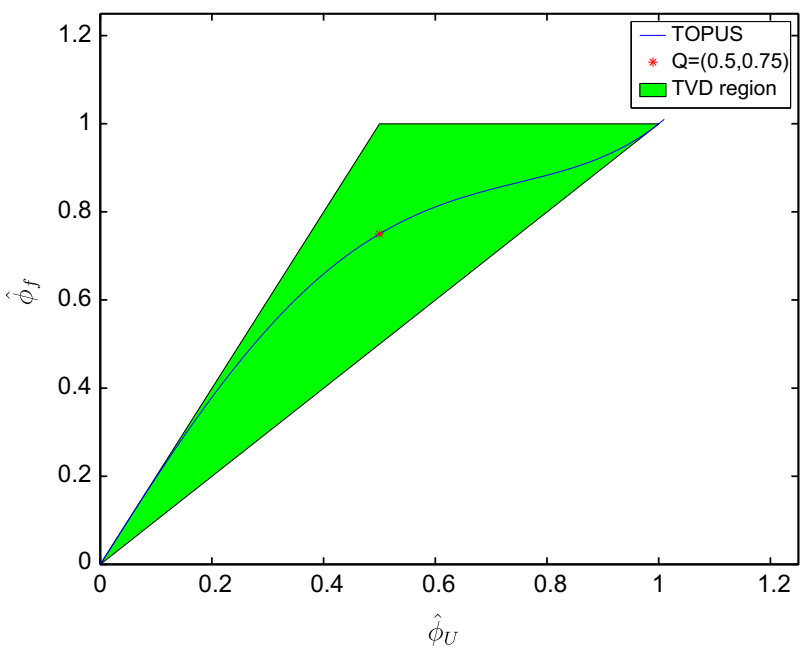

Fig. 2. TOPUS with $\alpha=2$ on TVD region.
Let $r_{f}$ be a local shock sensor satisfying Sweby's monotonicity preservation condition when $r_{f}$ tends to zero. Then the corresponding flux limiter $\psi=\psi\left(r_{f}\right)$ for the TOPUS scheme when $\alpha=2$ is deduced as follows. The variable $r_{f}$ is the ratio of upstream to downstream (consecutive) gradients

$r_{f}=\frac{\left(\frac{\partial \phi}{\partial x}\right)_{f}}{\left(\frac{\partial \phi}{\partial x}\right)_{g}}$,

which, for uniform meshes, can be rewritten as

$r_{f}=\frac{\phi_{U}-\phi_{R}}{\phi_{D}-\phi_{U}}$

and, in terms of the NV, expressed as

$r_{f}=\frac{\hat{\phi}_{U}}{1-\hat{\phi}_{U}}$

Consider the general approximation (FOU scheme plus an antidiffusive term) to the convected variable at the $f$ face

$\hat{\phi}_{f}=\hat{\phi}_{U}+\frac{1}{2} \psi\left(r_{f}\right)\left(1-\hat{\phi}_{U}\right)$.

From Eq. (4), with $\alpha=2$, Eqs. (7) and (8), one determines $\psi\left(r_{f}\right)$ as

$\psi\left(r_{f}\right)=\frac{\left(\left|r_{f}\right|+r_{f}\right)\left[3 r_{f}+1\right]}{\left(1+\left|r_{f}\right|\right)^{3}}$.

Fig. 3 displays the TOPUS flux limiter (9) in the $r_{f}-\psi\left(r_{f}\right)$ plane. It can be seen that the TOPUS flux limiter is a smooth function of $r_{f}$ (>0) (see Zijlema [76] and Piperno et al. [49]), so there would appear to be a real possibility that it might perform better than other well recognized TVD schemes.

It is important to recognize that the TOPUS upwind scheme developed here, for calculating flux derivatives, is derived from 1D theory; and in multidimensional cases, it has to be applied (following Zhang and Jackson [75]) to each of the coordinate directions separately.

\subsection{Implementation issues}

In this subsection, we show how TOPUS (and other upwinding schemes) may be incorporated into the discretized form of a number of model equations. In addition, a brief discussion concerning the stability of the computations and the choice of the CFL parameter is provided. In all calculations, for simplicity, first order

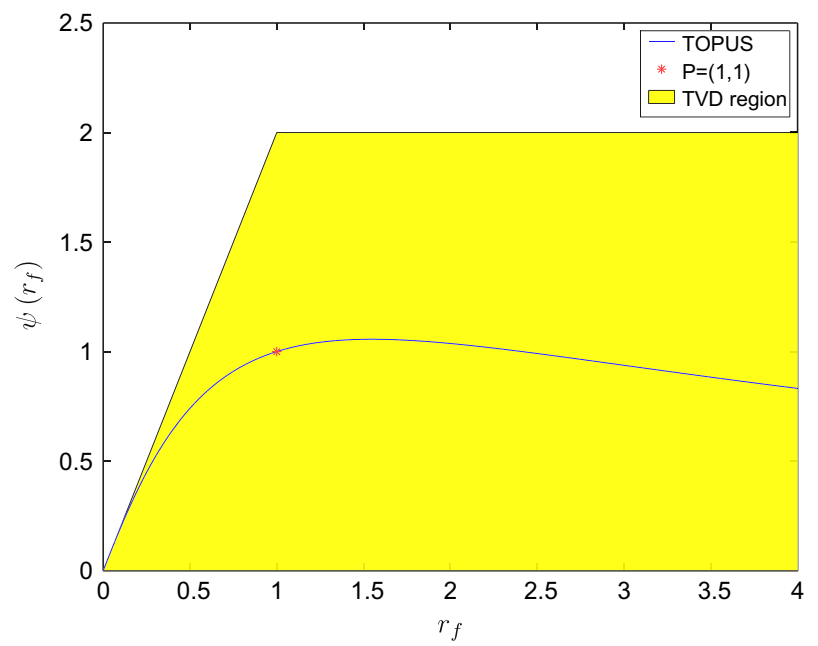

Fig. 3. Flux limiter $\psi\left(r_{f}\right)$ for the TOPUS with $\alpha=2$ on TVD region. 
explicit methods have been used for marching forward in time, except for the 1D inviscid Burgers equation, in which the 3th-order accurate explicit TVD Runge-Kutta method [61] has been used, and for the inviscid compressible flow over an airfoil, in which a second-order accurate, five-stage, explicit Runge-Kutta method [32] has been employed.

\subsubsection{The TOPUS scheme for $1 D$ and $2 D$ advection equation}

The advection of a quantity $\phi(x, t)$ by a convecting speed $a$ is modeled by the unsteady linear transport Eq. (1), where its exact solution is given by $\phi(x, t)=\phi_{0}(x-a t)$, with $\phi_{0}(x)$ the initial condition. Eq. (1) is one of the simplest one-dimensional convection models with a constant velocity contained within the initial data. Both varying the velocity fields and increasing the dimensions contribute to increased difficulties in modeling convection problems. But if a numerical scheme cannot solve the simple 1D case correctly, then it will be of little use in more complex situations. The solution is, in the 1D case, approximated by the method given by Eq. (2), where the convected variable $\phi$, at the $i-1 / 2$ and $i+1 / 2$ faces, is calculated using the upwind technology. Eq. (3) is then employed to transform Eq. (4), with $\alpha=2$, giving

$$
\phi_{i \pm 1 / 2}= \begin{cases}\phi_{R}+\left(\phi_{D}-\phi_{R}\right)\left[2\left(\hat{\phi}_{U}\right)^{4}-3\left(\hat{\phi}_{U}\right)^{3}+2 \hat{\phi}_{U}\right], & \hat{\phi}_{U} \in(0,1), \\ \phi_{U}, & \hat{\phi}_{U} \notin(0,1),\end{cases}
$$

with $D, U$ and $R$ previously defined. The solution for the 2D case follows similar lines.

The foregoing explicit numerical method has been implemented using an in-house computational fluid dynamics code, and its stability is governed by the CFL condition. In order to ensure stability, the time step is selected (automatically) in such a way that the CFL parameter satisfies CFL $\leqslant 1$. Here, CFL denotes, in the $1 \mathrm{D}$ case, the maximum propagation speed in a control volume at a given time level. In the $2 \mathrm{D}$ simulations, $\mathrm{CFL}=\max |u| \frac{\delta_{t}}{\delta_{x}}+$ $\max |v| \frac{\delta_{t}}{\delta_{x}}$.

\subsubsection{The TOPUS scheme for $1 D$ convection-diffusion equations}

We now consider a model of convection-diffusion, namely

$\frac{\partial \phi}{\partial t}+\varepsilon \frac{\partial \phi}{\partial x}=v \frac{\partial^{2} \phi}{\partial x^{2}}$

where the dependent variable $\phi$ may, for example, be considered to be a concentration in an incompressible fluid, $\varepsilon=\varepsilon(\phi)$ and $v$ is the diffusion coefficient. When in Eq. (11) $\varepsilon=1$, the model corresponds to a linear viscous flow model, with a boundary layer (see [22]). In the case when $\varepsilon=\frac{1}{2} \phi$, Eq. (11) becomes the nonlinear viscous Burgers' equation [14] with $v$ now interpreted as kinematic viscosity. It is well known that for large enough $v>0$, smooth solutions are obtained, and the energy of the system dissipates smoothly. However, for $v \rightarrow 0$, discontinuities (shocks) can develop in the solution, even for prescribed smooth initial data. Burgers' equation serves as a good model (combining nonlinear advection and linear diffusion) for understanding shock formation and turbulence. The numerical solution of this equation, in the linear case, is calculated in a manner similar to that used for solving the $1 \mathrm{D}$ advection equation in the previous subsection, with the diffusive term approximated by second order central differences. In the nonlinear case, the diffusive term is also approximated by the second order CD scheme, but the advection term (in the conservative form) is approximated by

$$
\left.\left(\varepsilon(\phi) \frac{\partial \phi}{\partial x}\right)\right|_{i}=\left.\frac{1}{2}\left(\frac{\partial \phi^{2}}{\partial x}\right)\right|_{i}=\frac{1}{2}\left(\frac{\bar{\phi}_{i+1 / 2} \cdot \phi_{i+1 / 2}-\bar{\phi}_{i-1 / 2} \cdot \phi_{i-1 / 2}}{2\left(\delta_{x} / 2\right)}\right),
$$

$\bar{\phi}_{i-1 / 2}=\frac{1}{2}\left(\phi_{i}+\phi_{i-1}\right) \quad$ and $\quad \bar{\phi}_{i+1 / 2}=\frac{1}{2}\left(\phi_{i+1}+\phi_{i}\right)$,

and the convected variable $\phi$ at the $i+1 / 2$ and $i-1 / 2$ calculed by the TOPUS scheme (10). It is important to observe here that by using the formula (13), for computing convection velocities, the simple formula (12), which provides a simplified implementation on the TOPUS, is rendered globally second-order accurate. However, in regions where the solution is sufficiently smooth, the order of convergence with the TOPUS scheme can be improved by implementating it in the context of flux function upwind reconstruction, associated with a high order time accuracy method and a suitable mesh refinement (similar to that appearing in Section 6, Eq. (84), of the Ref. [74]).

In the in-house numerical code equipped with the TOPUS scheme, the stability condition for solving Eq. (11) and the choice of the CFL parameter are made in a manner similar to the $1 \mathrm{D}$ advection equation.

\subsubsection{The TOPUS scheme for $1 D$ Euler equations}

The one-dimensional flow of an inviscid and compressible gas obeys the Euler equations (see, for example [32])

$\frac{\partial \mathbf{U}}{\partial t}+\frac{\partial \mathbf{F}(\mathbf{U})}{\partial x}=0$

where the conservative state vector $\mathbf{U}$ and the convective flux vector $\mathbf{F}(\mathbf{U})$ along the $x$-direction are defined by

$$
\left\{\begin{array}{l}
\mathbf{U}=[\rho, \rho u, E]^{T}, \\
\mathbf{F}=\left[\rho u, \rho u^{2}+p, u(E+p)\right]^{T} .
\end{array}\right.
$$

In the above vectors, $x$ is distance, $\rho$ density, $u$ the $x$-component of velocity, $p$ pressure, and $E$ the total energy per unit volume. The ratio of specific heats is set as $\gamma=1.4$ and, for a perfect gas, the system is completed by the equation of state $p=(\gamma-1)\left[E-\rho u^{2} / 2\right]$. Eq. (14) is numerically solved by using the explicit finite difference conservative formula

$\mathbf{U}_{i}^{n+1}=\mathbf{U}_{i}^{n}+\frac{\delta_{t}}{\delta_{x}}\left[\mathbf{F}_{i-1 / 2}-\mathbf{F}_{i+1 / 2}\right]^{n}$,

with the interface flux vector evaluated by using the method proposed by Roe and Pike [51], namely: first the Roe average values are computed (see Toro [62]); after that, for $k=1,2,3$, the eigenvalues $\tilde{\lambda}_{k}$ and the eigenvectors $\widetilde{K}^{(k)}$ of the Jacobian matrix $\widehat{A}$ (both evaluated using averaging) are computed; next the wave strengths $\widetilde{\alpha}_{k}$ are calculated; and then, with all of the aforementioned quantities, the flux vectors $\mathbf{F}_{i-1 / 2}$ and $\mathbf{F}_{i+1 / 2}$ (omitting for simplicity the time index) are directly approximated by using flux limiters in the framework of Sweby [60]. In particular, in a similar manner as was done by Hubbard and Garcia-Navarro [31], we implement the generic flux $\mathbf{F}_{i+1 / 2}$ as

$\mathbf{F}_{i+1 / 2}=\mathbf{F}_{i+1 / 2}^{\mathrm{LOW}}+\left.\frac{1}{2} \sum_{k=1}^{3} \operatorname{sign}\left(\tilde{\lambda}_{k}\right) \tilde{\lambda}_{k}\left(1-\left|\theta_{k}\right|\right) \psi\left(r_{f}^{k}\right) \tilde{\alpha}_{k} \widetilde{K}^{(k)}\right|_{i+1 / 2}$,

where $\mathbf{F}_{i+1 / 2}^{\mathrm{LOW}}$ is a monotone low-order accurate (building block) numerical flux, $\theta_{k}=\tilde{\lambda}_{k} \delta_{t} / \delta_{x}$, and $\psi(\cdot)$ is the TOPUS flux limiter (9). The sensor $r_{f}^{k}$ is calculated by

$r_{f}^{k}=\frac{\tilde{\alpha}_{k}^{\text {upwind }}}{\tilde{\alpha}_{k}^{\text {local }}}$,

where $\tilde{\alpha}_{k}^{\text {local }}=\left.\tilde{\alpha}_{k}\right|_{i+1 / 2}$ and $\tilde{\alpha}_{k}^{\text {upwind }}$ is obtained at the upwind location according to the velocity $\tilde{\lambda}_{k}$ at the face $i+1 / 2$. Finally, for the building block $\mathbf{F}_{i+1 / 2}^{\mathrm{LOW}}$ in Eq. (17) we use

$\mathbf{F}_{i+1 / 2}^{\mathrm{LOW}}=\frac{1}{2}\left(\mathbf{F}_{i}+\mathbf{F}_{i+1}\right)-\frac{1}{2} \sum_{k=1}^{3} \tilde{\alpha}_{k}\left|\tilde{\lambda}_{k}\right| \widetilde{K}^{(k)}$. 
The numerical method for solving 1D Euler equations is explicit, and its stability is governed by the CFL condition (see [10]). In this case, the time step $\delta_{t}$ is assumed to satisfy

$\frac{\delta_{t}}{\delta_{x}} \max \left\{\left|\lambda_{i+\frac{1}{2}}^{-}\right|,\left|\lambda_{i+\frac{1}{2}}^{+}\right|\right\} \leqslant 1$,

where $\lambda_{i+\frac{1}{2}}^{ \pm}$are the numerical acoustic waves associated with the numerical flux function.

\subsubsection{The TOPUS scheme for $2 D$ Euler equations}

The two-dimensional time-dependent Euler equations in conservation form are

$\frac{\partial \mathbf{U}}{\partial t}+\frac{\partial \mathbf{F}(\mathbf{U})}{\partial x}+\frac{\partial \mathbf{G}(\mathbf{U})}{\partial y}=0$

where the conservative state vector $\mathbf{U}$ and the convective flux vectors $\mathbf{F}(\mathbf{U})$ and $\mathbf{G}(\mathbf{U})$ along the $x$ - and $y$-directions, respectively, are defined by

$$
\left\{\begin{array}{l}
\mathbf{U}=[\rho, \rho u, \rho v, E]^{T}, \\
\mathbf{F}=\left[\rho u, \rho u^{2}+p, \rho u v,(E+p) u\right]^{T}, \\
\mathbf{G}=\left[\rho v, \rho u v, \rho v^{2}+p,(E+p) v\right]^{T}, \\
E=\frac{p}{(\gamma-1)}+\frac{1}{2} \rho\left(u^{2}+v^{2}\right) .
\end{array}\right.
$$

In Eqs. (20) and (21), $v$ is $y$-velocity component; other constants and variables have been defined previously.

Two codes, implemented in the context of standard finite volume methodology, were employed to solve the conservation law systems (20) and (21): the software package CLAWPACK (Conservation LAWs PACKage) of Leveque [42] and the code proposed by Bigarella [11]. The TOPUS scheme was applied only in the specific limiter routines of these codes. In particular, in the case of 2D aerodynamic applications (two-dimensional limiters), the ideas of Bigarella and Azevedo [12] were used to generalize the derivative ratios, allowing the usage of any $1 \mathrm{D}$ limiter. In summary, the limiter $\psi\left(r_{f}\right)$ is an extension of the work of Barth and Jespersen [8] and is given by

$\psi\left(r_{f}\right)=\frac{\left(\left|r_{f}\right|+r_{f}\right)\left[3 r_{f}+1\right]+\epsilon_{\text {LIM }}}{\left(1+\left|r_{f}\right|\right)^{3}+\epsilon_{\text {LIM }}}$,

where $\epsilon_{L I M}$ is a control parameter designed to prevent singularities (see [12]). The sensor $r_{f}$ corresponds to the ratio of the $f$-th volume given by

$r_{f}= \begin{cases}\text { num }^{+} / \text {den }, & \text { if den }>0, \\ \text { num }^{-} / \text {den, }, & \text { if den }<0, \\ 1, & \text { if den }=0 .\end{cases}$

In the above equation, num $^{ \pm}$and den are defined as (see $[12,11]$ )

$\operatorname{num}^{+}=\max \left(q_{i}, q_{f}\right)-q_{i} ; \quad \operatorname{num}^{-}=\min \left(q_{i}, q_{f}\right)-q_{i} ; \quad$ den $=\left(q_{i}\right)_{f}-q_{i}$,

with $q_{i}$ a variable associated with the volume $i, q_{f}$ a variable associated with the face $f$ of the volume, and $\left(q_{i}\right)_{f}$ a variable associated with the volume $i$ reconstructed at the $f$ face.

\subsubsection{The TOPUS scheme for $2 D$ incompressible fluid flows}

The conservation laws for time-dependent 2D incompressible fluid flow are the continuity and the momentum (Navier-Stokes) equations. In the Einstein index notation they are, respectively,

$$
\begin{aligned}
& \frac{\partial u_{i}}{\partial x_{i}}=0 \\
& \frac{\partial u_{i}}{\partial t}+\frac{\partial u_{i} u_{j}}{\partial x_{j}}=-\frac{\partial p}{\partial x_{i}}+\frac{1}{R e} \frac{\partial}{\partial x_{j}}\left(\frac{\partial u_{i}}{\partial x_{j}}\right)+\frac{1}{F r^{2}} g_{i}, \quad i=1,2
\end{aligned}
$$

where $t$ is the time, $x_{i}$ the Cartesian coordinates, $u_{i}$ the corresponding velocity components, $p$ the kinematic pressure, $g_{i}$ the components of the gravitational acceleration, and $R=U_{0} D / v$ and $F r=U_{0} / \sqrt{D|\mathbf{g}|}$, the Reynolds and Froude numbers, respectively. Here the usual Einstein summation convention is applied to repeated indices. The dependent variables in Eqs. (25) and (26) have been nondimensionalized by a characteristic velocity $U_{0}$, a length scale $D$ and a reference kinematic viscosity $v$. To simulate the flow problems modeled by Eqs. (25) and (26), the primitive variable Marker-And-Cell (MAC, Los Alamos) method was used: this is a special case of the projection method of Chorin [20] described by Harlow and Welch [30] (see also McKee et al. [45]). This finite difference method, defined on a staggered grid system, has been incorporated into the 2D version of the Freeflow code [16]. The MAC method uses massless marker particles, which are employed to indicate the fluid configuration showing which regions are occupied by fluid and which are empty. At each time step, the marker particles are moved to new positions using local fluid velocities.

For the spatial advection terms of the Navier-Stokes Eq. (26), the application of the TOPUS scheme is as follows. For the staggered grid used in this paper, a $f$ face for discretization can assume one of the following faces of the control volume depicted in Fig. 4:

$$
\left(i+\frac{1}{2}, j\right) \text { or }\left(i, j+\frac{1}{2}\right) \text {. }
$$

The convected variable $\phi$, calculated by the TOPUS scheme, can be one of the velocity components $u$ or $v$. For conciseness, only the discretization of the nonlinear advection terms in the $u$-component of the Navier-Stokes equations will be presented. The discretization of the other nonlinear terms are similar. In the position $\left(i+\frac{1}{2}, j\right)$ of the 2D computational mesh (see Fig. 4 ), this term can be approximated by the following conservative scheme:

$$
\begin{aligned}
\left.\left(\frac{\partial(u u)}{\partial x}+\frac{\partial(u v)}{\partial y}\right)\right|_{i+\frac{1}{2}, j} \approx & \frac{\bar{u}_{i+1, j} \cdot u_{i+1, j}-\bar{u}_{i, j} \cdot u_{i, j}}{\delta_{x}} \\
& +\frac{\bar{v}_{i+\frac{1}{2}, j+\frac{1}{2}} \cdot u_{i+\frac{1}{2}, j+\frac{1}{2}}-\bar{v}_{i+\frac{1}{2}, j-\frac{1}{2}} \cdot u_{i+\frac{1}{2}, j-\frac{1}{2}}}{\delta_{y}}
\end{aligned}
$$

where the advection velocities $\bar{u}_{i+1, j}, \bar{u}_{i, j}, \bar{v}_{i+\frac{1}{2}, j+\frac{1}{2}}$ and $\bar{v}_{i+\frac{1}{2}, j-\frac{1}{2}}$ are obtained by averaging, in a similar manner as in Eq. (13) for Burgers' equation, and the convected velocities follow similar procedures to that in Eq. (12). The following criterion was used for selecting an appropriate time step

$\delta_{t}=\min \left\{F A C T_{1} \cdot \delta_{t \mathrm{CFL}}, F A C T_{2} \cdot \delta_{t \mathrm{VISC}}\right\}$,

where $0<F A C T_{1} \leqslant 1$ and $0<F A C T_{2} \leqslant 1$ are constants chosen to ensure that the calculations are stable with

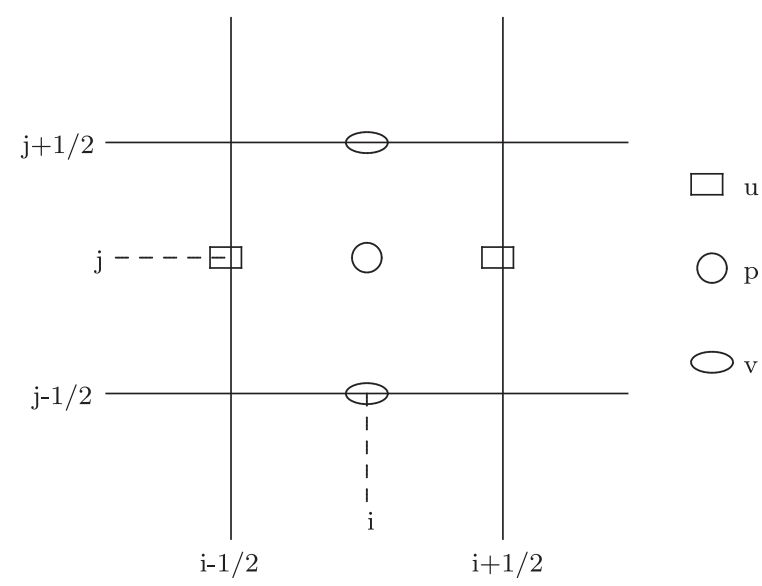

Fig. 4. Cell-variable locations for $2 \mathrm{D}$ calculation, showing the faces where $u$ and $v$ velocities are evaluated. 

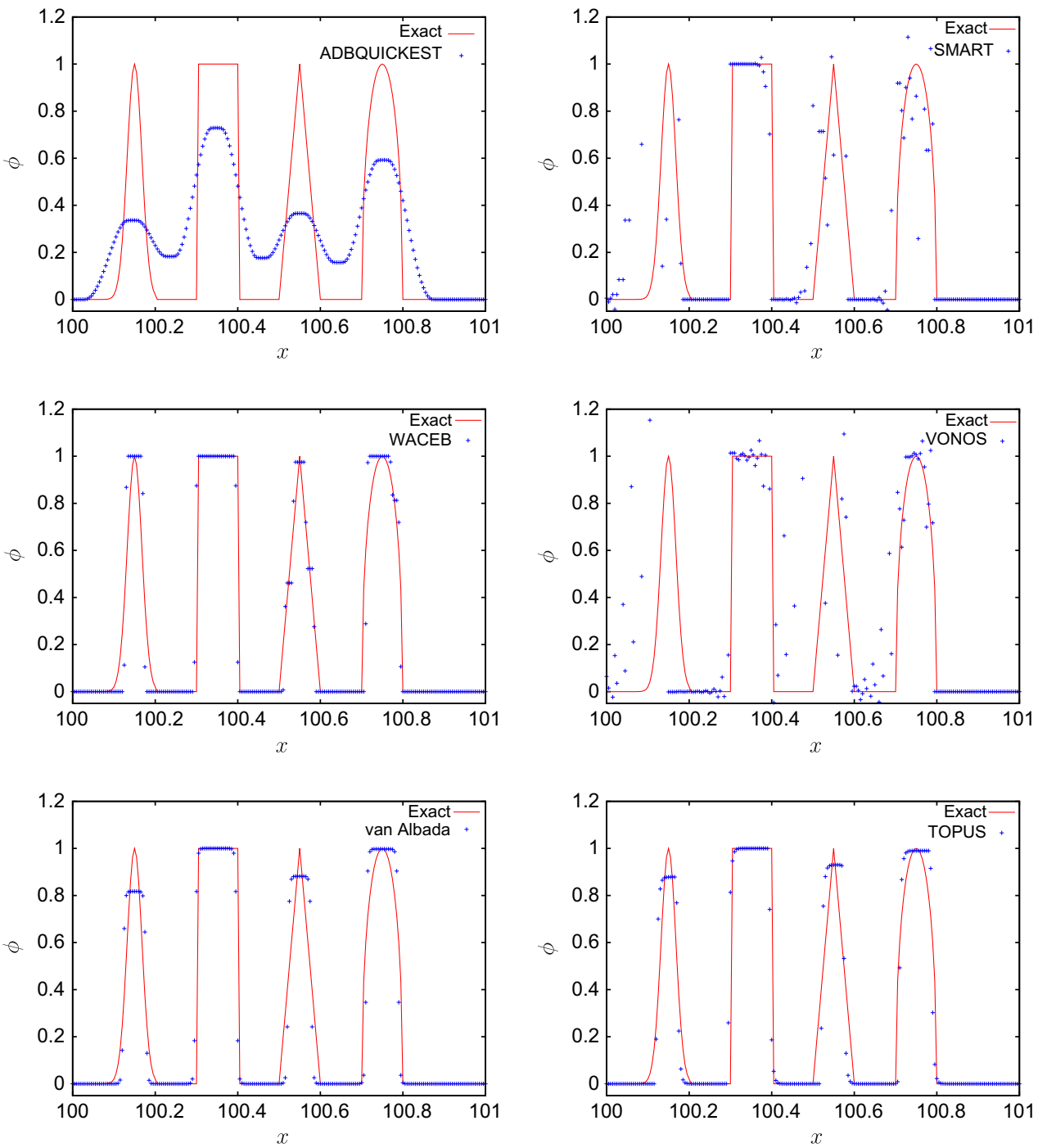

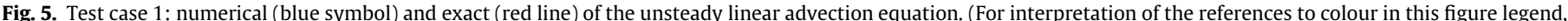
the reader is referred to the web version of this article.)

Table 1

Computing time per mesh point per iteration and normalized costs by the unit cost of the WACEB scheme (cheapest).

\begin{tabular}{lll}
\hline Scheme & Unit cost $(\mu \mathrm{s})$ & Normalized costs \\
\hline ADBQUICKEST & 0.35 & 1.13 \\
SMART & 2.13 & 6.87 \\
WACEB & 0.31 & 1.00 \\
VONOS & 1.45 & 4.68 \\
van Albada & 0.33 & 1.06 \\
TOPUS & 0.32 & 1.03 \\
\hline
\end{tabular}

$\delta_{t \mathrm{CFL}}=\max \left\{\frac{\delta_{x}}{|u|}, \frac{\delta_{y}}{|v|}\right\} \quad$ and $\quad \delta_{t \mathrm{VISC}}=\frac{\operatorname{Re}}{2} \frac{\delta_{x}^{2} \delta_{y}^{2}}{\delta_{x}^{2}+\delta_{y}^{2}}$

\subsubsection{Popular upwinding schemes}

In this subsection, we list the schemes that are to be compared with TOPUS. We also detail flux limiter functions.Non-normalized variable schemes:

- SMART [24]:

$$
\phi_{f}= \begin{cases}\phi_{U} & \text { if } \hat{\phi}_{U} \notin[0,1], \\ 10 \phi_{U}-9 \phi_{R} & \text { if } 0 \leqslant \hat{\phi}_{U}<3 / 74, \\ \frac{1}{8}\left(3 \phi_{D}+6 \phi_{U}-\phi_{R}\right) & \text { if } 3 / 74 \leqslant \hat{\phi}_{U}<5 / 6, \\ \phi_{D} & \text { if } 5 / 6 \leqslant \hat{\phi}_{U} \leqslant 1 ;\end{cases}
$$

- VONOS [65]:

$$
\phi_{f}= \begin{cases}\phi_{U} & \text { if } \hat{\phi}_{U} \notin[0,1] \\ 10 \phi_{U}-9 \phi_{R} & \text { if } 0 \leqslant \hat{\phi}_{U}<3 / 74 \\ \frac{3}{8} \phi_{D}+\frac{3}{4} \phi_{U}-\frac{1}{8} \phi_{R} & \text { if } 3 / 74 \leqslant \hat{\phi}_{U}<1 / 2 \\ 1.5 \phi_{U}-0.5 \phi_{R} & \text { if } 1 / 2 \leqslant \hat{\phi}_{U}<2 / 3 \\ \phi_{D} & \text { if } 2 / 3 \leqslant \hat{\phi}_{U} \leqslant 1\end{cases}
$$

- WACEB [57]:

$$
\phi_{f}= \begin{cases}\phi_{U} & \text { if } \hat{\phi}_{U} \notin[0,1], \\ 2 \phi_{U}-\phi_{R} & \text { if } 0 \leqslant \hat{\phi}_{U}<3 / 10, \\ \frac{3}{4} \phi_{U}-\frac{3}{8} \phi_{D}-\frac{1}{8} \phi_{R} & \text { if } 3 / 10 \leqslant \hat{\phi}_{U}<5 / 6, \\ \phi_{D} & \text { if } 5 / 6 \leqslant \hat{\phi}_{U} \leqslant 1\end{cases}
$$


Table 2

Errors and computed convergence rates for $2 \mathrm{D}$ advection equation, with $\mathrm{CFL}=0.5$ at time $t=2$. Here, ADB refers to ADBQUICKEST.

\begin{tabular}{|c|c|c|c|c|c|}
\hline Scheme & Mesh & $L_{1}$-error & Convergence rate & $L_{2}$-error & Convergence rate \\
\hline ADB & $\begin{array}{l}16 \times 16 \\
32 \times 32 \\
64 \times 64 \\
128 \times 128 \\
256 \times 256\end{array}$ & $\begin{array}{l}7.40 \mathrm{e}-3 \\
1.81 \mathrm{e}-3 \\
4.74 \mathrm{e}-4 \\
1.20 \mathrm{e}-4 \\
3.03 \mathrm{e}-5\end{array}$ & $\begin{array}{l}- \\
2.0 \\
1.9 \\
2.0 \\
2.0\end{array}$ & $\begin{array}{c}3.00 \mathrm{e}-2 \\
1.09 \mathrm{e}-2 \\
4.14 \mathrm{e}-3 \\
1.50 \mathrm{e}-3 \\
5.37 \mathrm{e}-4\end{array}$ & $\begin{array}{l}- \\
1.5 \\
1.4 \\
1.5 \\
1.5\end{array}$ \\
\hline Superbee & $\begin{array}{l}16 \times 16 \\
32 \times 32 \\
64 \times 64 \\
128 \times 128 \\
256 \times 256\end{array}$ & $\begin{array}{l}8.94 \mathrm{e}-3 \\
1.87 \mathrm{e}-3 \\
4.60 \mathrm{e}-4 \\
1.19 \mathrm{e}-4 \\
3.02 \mathrm{e}-5\end{array}$ & $\begin{array}{l}- \\
2.3 \\
2.0 \\
2.0 \\
2.0\end{array}$ & $\begin{array}{l}3.63 e-2 \\
1.15 e-2 \\
4.05 e-3 \\
1.49 e-3 \\
5.35 e-4\end{array}$ & $\begin{array}{l}- \\
1.7 \\
1.5 \\
1.4 \\
1.5\end{array}$ \\
\hline van Leer & $\begin{array}{l}16 \times 16 \\
32 \times 32 \\
64 \times 64 \\
128 \times 128 \\
256 \times 256\end{array}$ & $\begin{array}{l}6.97 e-3 \\
1.82 e-3 \\
4.74 e-4 \\
1.20 e-4 \\
3.03 e-5\end{array}$ & $\begin{array}{l}- \\
1.9 \\
1.9 \\
2.0 \\
2.0\end{array}$ & $\begin{array}{l}2.81 \mathrm{e}-2 \\
1.09 \mathrm{e}-2 \\
4.14 \mathrm{e}-3 \\
1.50 \mathrm{e}-3 \\
5.37 \mathrm{e}-4\end{array}$ & $\begin{array}{l}- \\
1.4 \\
1.4 \\
1.5 \\
1.5\end{array}$ \\
\hline van Albada & $\begin{array}{l}16 \times 16 \\
32 \times 32 \\
64 \times 64 \\
128 \times 128 \\
256 \times 256\end{array}$ & $\begin{array}{l}8.85 e-3 \\
4.47 e-3 \\
6.08 e-4 \\
1.46 e-4 \\
3.40 e-5\end{array}$ & $\begin{array}{l}- \\
1.0 \\
2.9 \\
2.1 \\
2.1\end{array}$ & $\begin{array}{l}3.61 \mathrm{e}-2 \\
2.67 \mathrm{e}-2 \\
5.29 \mathrm{e}-3 \\
1.82 \mathrm{e}-3 \\
6.02 \mathrm{e}-4\end{array}$ & $\begin{array}{l}- \\
0.4 \\
2.3 \\
1.5 \\
1.6\end{array}$ \\
\hline TOPUS & $\begin{array}{l}16 \times 16 \\
32 \times 32 \\
64 \times 64 \\
128 \times 128 \\
256 \times 256\end{array}$ & $\begin{array}{l}1.01 \mathrm{e}-2 \\
3.39 \mathrm{e}-3 \\
5.63 \mathrm{e}-4 \\
1.34 \mathrm{e}-4 \\
3.20 \mathrm{e}-5\end{array}$ & $\begin{array}{l}- \\
1.6 \\
2.6 \\
2.1 \\
2.1\end{array}$ & $\begin{array}{l}4.09 \mathrm{e}-2 \\
2.02 \mathrm{e}-2 \\
4.91 \mathrm{e}-3 \\
1.67 \mathrm{e}-3 \\
5.66 \mathrm{e}-4\end{array}$ & $\begin{array}{l}- \\
1.0 \\
2.0 \\
1.6 \\
1.6\end{array}$ \\
\hline
\end{tabular}

- Superbee [7]:

$$
\phi_{f}= \begin{cases}\phi_{U} & \text { if } \hat{\phi}_{U} \notin[0,1], \\ 2 \phi_{U}-\phi_{R} & \text { if } 0 \leqslant \hat{\phi}_{U}<1 / 3, \\ \frac{1}{2}\left(\phi_{D}+\phi_{U}\right) & \text { if } 1 / 3 \leqslant \hat{\phi}_{U}<1 / 2, \\ \frac{3}{2} \phi_{U}-\frac{1}{2} \phi_{R} & \text { if } 1 / 2 \leqslant \hat{\phi}_{U}<2 / 3, \\ \phi_{D} & \text { if } 2 / 3 \leqslant \hat{\phi}_{U} \leqslant 1\end{cases}
$$

$$
\begin{aligned}
& \alpha_{D}=\frac{1}{6}\left(2-3 \theta+\theta^{2}\right), \quad \alpha_{U}=\frac{1}{6}\left(5+3 \theta-2 \theta^{2}\right), \quad \alpha_{R}=\frac{1}{6}\left(1-\theta^{2}\right), \\
& a=\frac{2-3 \theta+\theta^{2}}{7-9 \theta+2 \theta^{2}}, \quad b=\frac{-4+3 \theta+\theta^{2}}{-5+3 \theta+2 \theta^{2}} .
\end{aligned}
$$

Flux limiter functions:

- Minmod [28]: $\psi\left(r_{f}\right)=\operatorname{minmod}\left(1, r_{f}\right)$;

- Superbee [7]: $\psi\left(r_{f}\right)=\max \left(0, \min \left(1,2 r_{f}\right), \min \left(2, r_{f}\right)\right)$;

- monotonized centered (MC) [67]: $\psi\left(r_{f}\right)=\max \left(0, \min \left(\left(1+r_{f}\right)\right)\right.$ $\left.2,2,2 r_{f}\right)$ );

$$
\phi_{f}= \begin{cases}\phi_{U} & \text { if } \hat{\phi}_{U} \notin[0,1], \\ \frac{7}{4} \phi_{U}-\frac{3}{4} \phi_{R} & \text { if } 0 \leqslant \hat{\phi}_{U}<3 / 8, \\ \frac{3}{8} \phi_{D}-\frac{3}{4} \phi_{U}-\frac{1}{8} \phi_{R} & \text { if } 3 / 8 \leqslant \hat{\phi}_{U}<3 / 4, \\ \frac{3}{4} \phi_{D}+\frac{1}{4} \phi_{U} & \text { if } 3 / 4 \leqslant \hat{\phi}_{U} \leqslant 1 ;\end{cases}
$$

- ADBQUICKEST [23]:

$$
\phi_{f}= \begin{cases}\phi_{U} & \text { if } \hat{\phi}_{U} \notin[0,1], \\ (2-\theta) \phi_{U}-(1-\theta) \phi_{R} & \text { if } 0 \leqslant \hat{\phi}_{U}<a, \\ \left.\alpha_{D} \phi_{D}+\alpha_{U} \phi_{U}-\alpha_{R} \phi_{R}\right) & \text { if } a \leqslant \hat{\phi}_{U} \leqslant b, \\ (1-\theta) \phi_{D}+\theta \phi_{U} & \text { if } b<\hat{\phi}_{U}<1 ;\end{cases}
$$

\begin{tabular}{|c|c|c|c|c|c|}
\hline Scheme & Mesh & $L_{1}$-error & Convergence rate & $L_{\infty}$-error & Convergence rate \\
\hline \multirow[t]{5}{*}{ van Albada } & 20 & $2.880 \mathrm{e}-02$ & - & $7.017 e-02$ & - \\
\hline & 40 & $7.872 \mathrm{e}-03$ & 1.8 & $2.462 \mathrm{e}-02$ & 1.5 \\
\hline & 80 & $1.700 \mathrm{e}-03$ & 2.2 & $9.010 \mathrm{e}-03$ & 1.4 \\
\hline & 160 & $5.350 \mathrm{e}-04$ & 1.7 & $2.862 \mathrm{e}-03$ & 1.6 \\
\hline & 320 & $1.248 \mathrm{e}-04$ & 2.1 & $8.219 \mathrm{e}-04$ & 1.8 \\
\hline \multirow[t]{5}{*}{ TOPUS } & 20 & $1.825 \mathrm{e}-02$ & - & $5.250 \mathrm{e}-02$ & - \\
\hline & 40 & $3.551 \mathrm{e}-03$ & 2.3 & $1.530 \mathrm{e}-02$ & 1.7 \\
\hline & 80 & $9.460 \mathrm{e}-04$ & 1.9 & $4.630 \mathrm{e}-03$ & 1.7 \\
\hline & 160 & $1.720 \mathrm{e}-04$ & 2.4 & $1.150 \mathrm{e}-03$ & 2.0 \\
\hline & 320 & $3.492 \mathrm{e}-05$ & 2.3 & $3.303 e-04$ & 1.8 \\
\hline
\end{tabular}

with

Table 3

Errors and computed convergence rates for 1D inviscid Burgers' equation, with $u_{0}(x)=1+0.5 \sin (\pi x),-1<x<1$ at time $t=0.12$.
In order to demonstrate the behavior, validity, flexibility, robustness and practicality of the TOPUS scheme, we have performed numerous simulations based on benchmark test cases, including 2D compressible/incompressible flows. Comparisons are made both with exact solutions and with well-recognized 

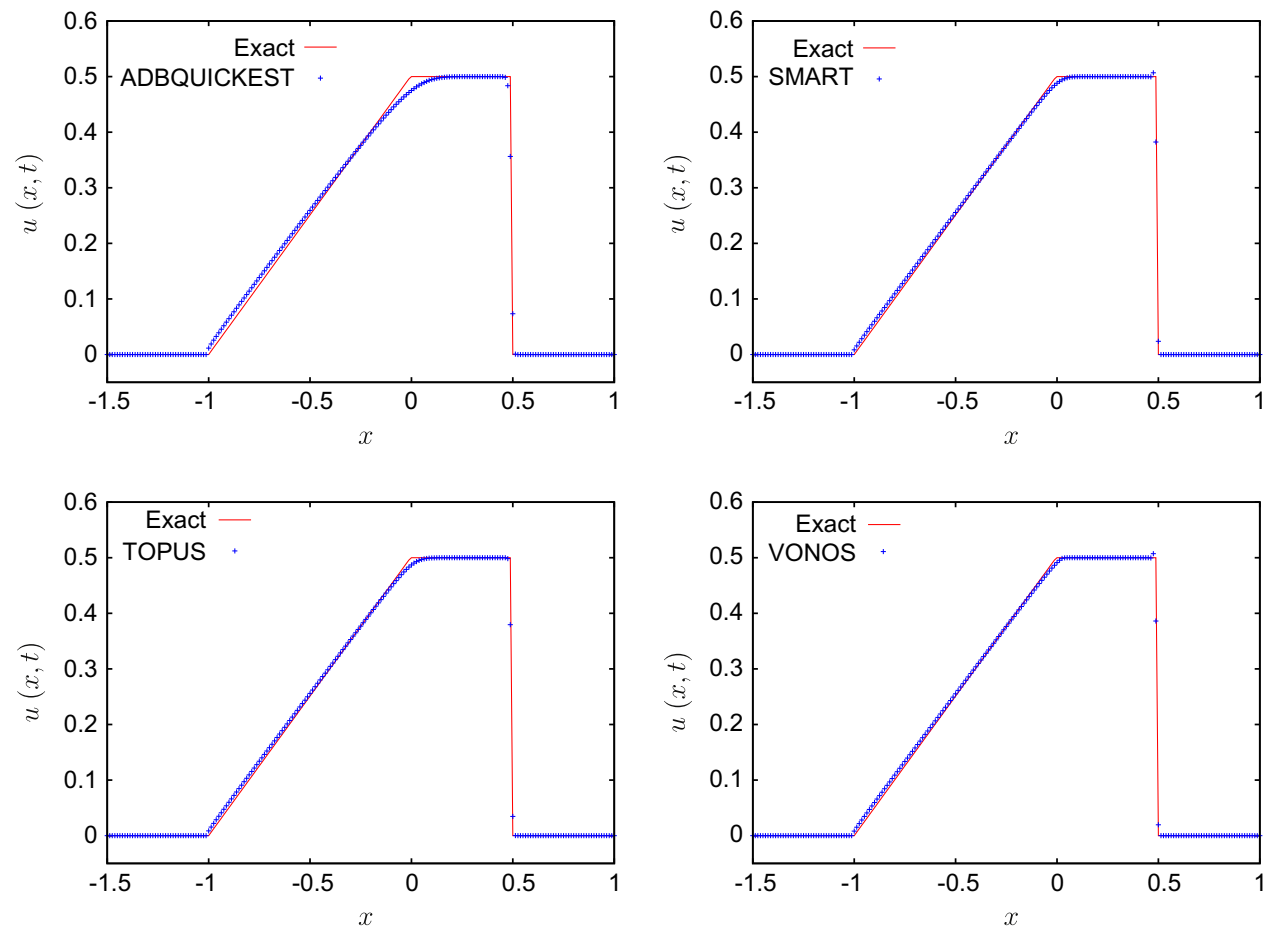

Fig. 6. Solution of the Riemann problem for the inviscid Burgers' equation using the initial condition (29).

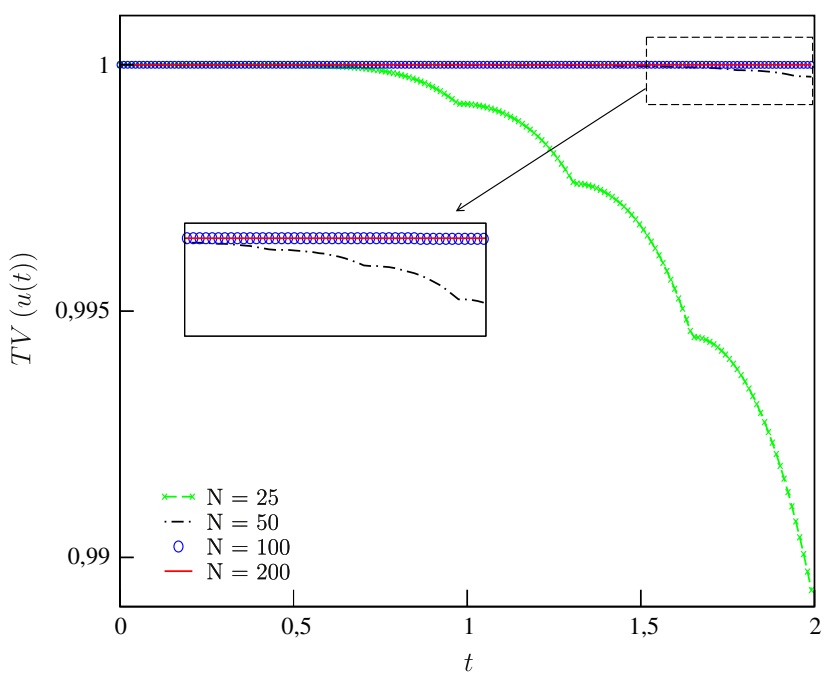

Fig. 7. Total variation of the Burgers' equation with respect to time.

high-resolution bounded schemes. The formal order of accuracy of the schemes is 2, except for TOPUS, ADBQUICKEST, WACEB, SMART and 3rd-WENO schemes. For the second order spatial derivatives and pressure terms, second order CD was employed. In all numerical tests presented in this article, the TOPUS scheme is used with $\alpha=2$. For compressible flow calculations, the parameter $\epsilon_{L I M}$ (appearing in Eq. (22)) is set as $10^{-7}$. All simulations have been performed on a Sony VAIO VGN-CS325J laptop with a Intel Core 2 Duo T6500/ $2.1 \mathrm{GHz}$ (Dual-Core) processor and 4 Gbytes RAM running Linux 2.6.30-bpo.2-amd64.

\subsection{D scalar advection problem}

The first test case consists of the advection of a quantity $\phi(x, t)$ with a convecting speed $a=1$ modeled by the unsteady linear advection Eq. (1). The physical relevance of this problem is that it models entropy waves in gas dynamics. In this test case, Eq. (1), $x \in[0,101]$, is solved in conjunction with the following combination of smooth and sharp distributions as initial condition:

$$
-1 \phi_{0}(x)= \begin{cases}e^{-\log 50\left(\frac{x-0.15}{0.05}\right)^{2}}, & x \in[0,0.2), \\ 1, & x \in(0.3,0.4), \\ 20 x-10, & x \in(0.5,0.55), \\ 12-20 x, & x \in[0.55,0.66), \\ \sqrt{1-\left(\frac{x-0.75}{0.05}\right)^{2}}, & x \in(0.7,0.8], \\ 0, & \text { otherwise. }\end{cases}
$$

In the foregoing numerical simulations, a mesh size of $N=2200$ computational cells was adopted with time spacing $\delta_{t}=0.0025$ and the final simulation time $t=100.0$. Fig. 5 depicts the exact solution and the numerical results obtained with ADBQUICKEST, SMART, WACEB, VONOS, van Albada and TOPUS schemes. It can be seen from this figure that, for long simulation times, WACEB, van Albada and TOPUS schemes perform reasonably well, but exhibit the peak "clipping" problem.

The unit costs (computation time per mesh point per iteration) and the unit normalized costs for the various choice of limiters are provided in Table 1 . The cost for the TOPUS scheme is smaller than the corresponding costs for SMART, VONOS, ADBQUICKEST and van Albada schemes, but higher than that for WACEB scheme. The computations were then carried out on a sequence of meshes and similar patterns were observed.

\section{2. $2 \mathrm{D}$ scalar advection problem}

We choose the 2D scalar convection equation, on the unit square, to check the numerical order of accuracy of the TOPUS scheme, with the advection velocities $u=v=1$, the initial condition $\phi_{0}(x, y)=\sin (2 \pi x) \sin (2 \pi y)$

and with periodic boundary conditions. The exact solution is given by (see [78]) 
Table 4

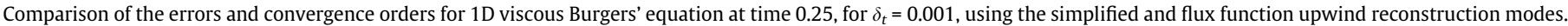
Measured errors as function of the mesh size and $R e=20$.

\begin{tabular}{|c|c|c|c|c|c|c|c|}
\hline Mode & Mesh & $L_{1}$-error & Order & $L_{2}$-error & Order & $L_{\infty}$-error & Order \\
\hline \multirow[t]{4}{*}{ Simplified } & 25 & $9.374 \mathrm{e}-4$ & - & $1.696 \mathrm{e}-3$ & - & $4.035 \mathrm{e}-3$ & - \\
\hline & 50 & $3.045 \mathrm{e}-4$ & 1.6 & $5.012 \mathrm{e}-4$ & 1.8 & $1.089 \mathrm{e}-3$ & 1.9 \\
\hline & 100 & $9.111 \mathrm{e}-5$ & 1.7 & $1.400 \mathrm{e}-4$ & 1.8 & $2.887 e-4$ & 1.9 \\
\hline & 200 & $2.472 \mathrm{e}-5$ & 1.8 & $3.700 e-5$ & 1.9 & $7.420 \mathrm{e}-5$ & 2.0 \\
\hline \multirow[t]{4}{*}{ Flux function } & 25 & $2.772 \mathrm{e}-3$ & - & $3.574 \mathrm{e}-3$ & - & $5.729 \mathrm{e}-3$ & - \\
\hline & 50 & $5.595 \mathrm{e}-4$ & 2.3 & $7.360 \mathrm{e}-4$ & 2.3 & $1.228 \mathrm{e}-3$ & 2.2 \\
\hline & 100 & $9.544 \mathrm{e}-5$ & 2.6 & $1.222 \mathrm{e}-4$ & 2.6 & $2.238 \mathrm{e}-4$ & 2.5 \\
\hline & 200 & $1.397 e-5$ & 2.8 & $1.797 \mathrm{e}-5$ & 2.8 & $3.374 \mathrm{e}-5$ & 2.7 \\
\hline
\end{tabular}

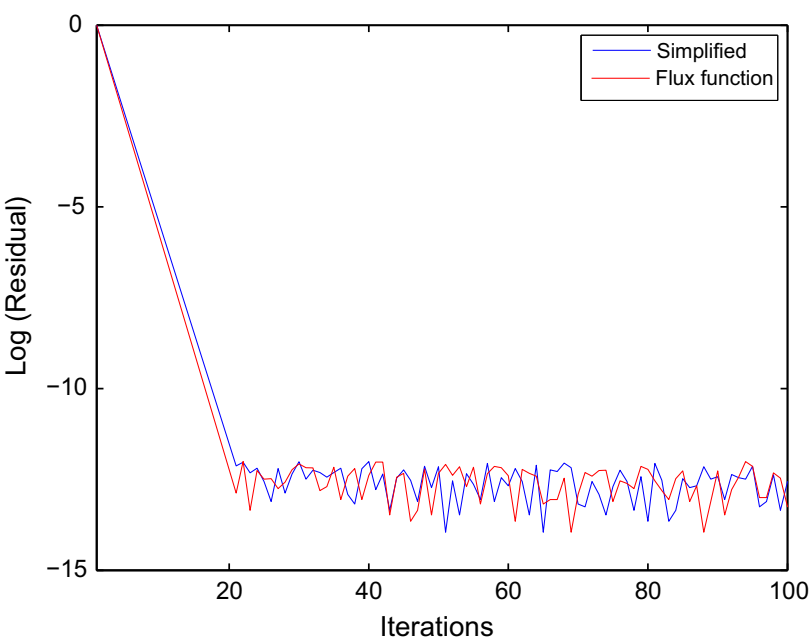

Fig. 8. Convergence history obtained with the TOPUS scheme, implemented in the context of simplified and flux function upwind reconstruction modes, for the 1D viscous Burgers' equation at $\mathrm{Re}=20$.

$\phi(x, y, t)=\sin (2 \pi(x-t)) \sin (2 \pi(y-t))$.

Table 2 gives the $L_{1}$ and $L_{2}$ errors and the corresponding orders of convergence for the ADBQUICKEST, Superbee, TOPUS, van Albada and van Leer schemes with $C F L=0.5$ at final time $t=2$. Practically, the same order of convergence is observed for all schemes. The numerical results are omitted here to save space.

\subsection{D Burgers' equation}

Here simulations are performed for the classic 1D Burgers' equation, namely Eq. (11) with $\phi=u$ and $\epsilon=\frac{1}{2} u$. Both the inviscid $(v=0.0)$ and viscous $(v=0.05)$ cases are considered. Firstly, we solve the inviscid case with a smooth initial distribution to study the convergence. Next, we employ a specific initial distribution to assess the shock capturing capabilities of the schemes. We address, in the following, the nonlinear stability of the TOPUS scheme. Finally, by resolving the viscous case, we check the impact of the flux function upwind reconstruction on the TOPUS's convergence rate.

The accuracy of the spatial discretization is checked by solving Eq. (11), $x \in[-1,1]$, with the smooth initial distribution $u(x, 0)=1+0.5 \sin (\pi x)$. The third order accurate TVD Runge-Kutta method presented in Tang and Warnecke [61] was used for evolution in time. The accuracy for all the popular upwinding schemes, given in Section 2.2.6, and that for Shu and Osher's third-order WENO are shown to be $O\left(h^{5 / 2}\right)$ in both the discrete $L_{1}$ and $L_{\infty}$ norms. The FOU scheme using a mesh size of 800 cells has been used for determining errors. In particular, Table 3 summarizes the errors and convergence rates observed for $u$ at time $t=0.12$ for the TOPUS and van Albada schemes. One can see that, for this nonlinear test case, convergence rates in excess of second order is obtained several times with the TOPUS scheme. One possible reason for these rates may be the rapid dampening of oscillations in the computed variable $u$ as the mesh increases.

The shock capturing property of the TOPUS scheme is studied by solving (11) with the initial distribution

Table 5

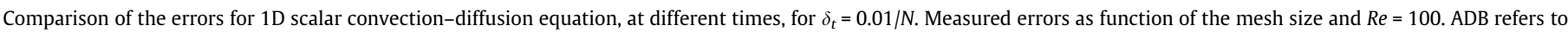
ADBQUICKEST

\begin{tabular}{|c|c|c|c|c|c|c|c|}
\hline Scheme & $N$ & $L_{1}$-error & Order & $L_{2}$-error & Order & $L_{\infty}$-error & Order \\
\hline \multirow[t]{4}{*}{ ADB } & 80 & $3.748 \mathrm{e}-2$ & - & $4.349 \mathrm{e}-2$ & - & $5.545 \mathrm{e}-2$ & - \\
\hline & 160 & $6.810 \mathrm{e}-3$ & 2.5 & $1.013 \mathrm{e}-2$ & 2.1 & $1.379 \mathrm{e}-2$ & 2.0 \\
\hline & 320 & $2.700 \mathrm{e}-3$ & 1.3 & $4.497 e-3$ & 1.2 & $6.299 \mathrm{e}-3$ & 1.1 \\
\hline & 640 & $9.200 \mathrm{e}-4$ & 1.6 & $1.645 \mathrm{e}-3$ & 1.5 & $2.311 \mathrm{e}-3$ & 1.4 \\
\hline \multirow[t]{4}{*}{ SMART } & 80 & $5.960 \mathrm{e}-3$ & - & $6.731 \mathrm{e}-3$ & - & $8.284 \mathrm{e}-3$ & - \\
\hline & 160 & $1.430 \mathrm{e}-3$ & 2.1 & $2.122 \mathrm{e}-3$ & 1.7 & $2.877 e-3$ & 1.5 \\
\hline & 320 & $8.600 \mathrm{e}-4$ & 0.7 & $1.437 \mathrm{e}-3$ & 0.6 & $2.005 e-3$ & 0.5 \\
\hline & 640 & $2.900 \mathrm{e}-4$ & 1.6 & $5.220 \mathrm{e}-4$ & 1.5 & $7.334 \mathrm{e}-4$ & 1.5 \\
\hline \multirow[t]{4}{*}{ Superbee } & 80 & $3.647 \mathrm{e}-2$ & - & $4.228 \mathrm{e}-2$ & - & $5.386 e-2$ & - \\
\hline & 160 & $1.212 \mathrm{e}-2$ & 1.6 & $1.793 e-2$ & 1.2 & $2.404 \mathrm{e}-2$ & 1.2 \\
\hline & 320 & $3.020 \mathrm{e}-3$ & 2.0 & $4.998 \mathrm{e}-3$ & 1.8 & $6.970 \mathrm{e}-3$ & 1.8 \\
\hline & 640 & $7.500 \mathrm{e}-4$ & 2.0 & $1.335 \mathrm{e}-3$ & 1.9 & $1.888 \mathrm{e}-3$ & 1.9 \\
\hline \multirow[t]{4}{*}{ van Albada } & 80 & $8.023 e-2$ & - & $9.582 \mathrm{e}-2$ & - & $1.266 \mathrm{e}-1$ & - \\
\hline & 160 & $2.607 e-2$ & 1.6 & $3.912 \mathrm{e}-2$ & 1.3 & $5.401 \mathrm{e}-2$ & 1.2 \\
\hline & 320 & $6.830 \mathrm{e}-3$ & 1.9 & $1.140 \mathrm{e}-2$ & 1.8 & $1.601 \mathrm{e}-2$ & 1.8 \\
\hline & 640 & $1.630 \mathrm{e}-3$ & 2.1 & $2.923 \mathrm{e}-3$ & 2.0 & $4.122 \mathrm{e}-3$ & 2.0 \\
\hline \multirow[t]{4}{*}{ TOPUS } & 80 & $5.330 \mathrm{e}-2$ & - & $6.248 \mathrm{e}-2$ & - & $8.073 e-2$ & - \\
\hline & 160 & $1.319 \mathrm{e}-2$ & 2.0 & $1.968 \mathrm{e}-2$ & 1.7 & $2.692 \mathrm{e}-2$ & 1.6 \\
\hline & 320 & $2.690 \mathrm{e}-3$ & 2.3 & $4.484 \mathrm{e}-3$ & 2.1 & $6.278 \mathrm{e}-3$ & 2.1 \\
\hline & 640 & $5.300 \mathrm{e}-4$ & 2.3 & $9.608 \mathrm{e}-4$ & 2.2 & $1.352 \mathrm{e}-3$ & 2.2 \\
\hline
\end{tabular}



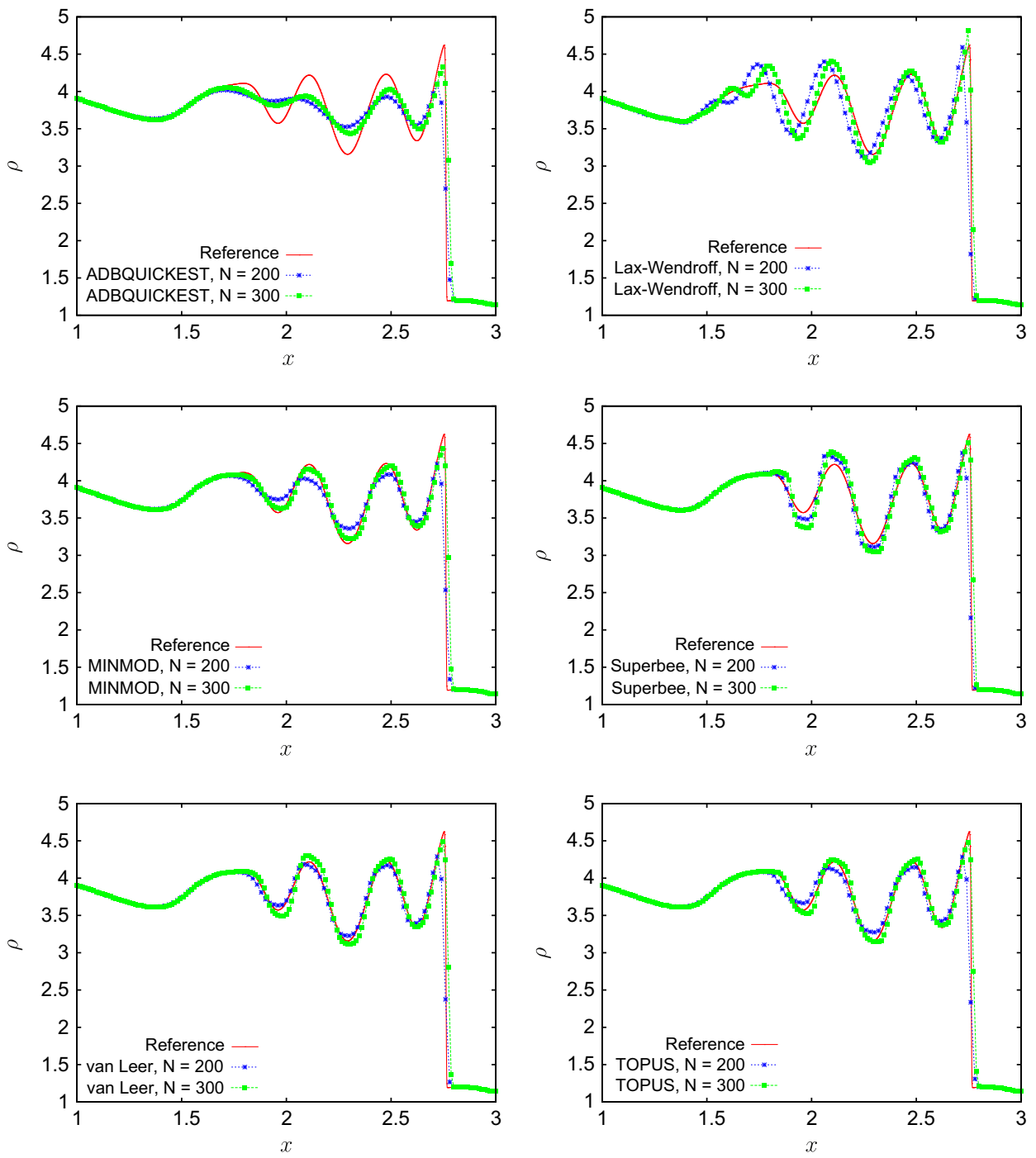

Fig. 9. Density distribution for the Shu-Osher shock tube problem using ADBQUICKEST, Lax-Wendroff, Minmod, Superbee, van Leer and TOPUS schemes.

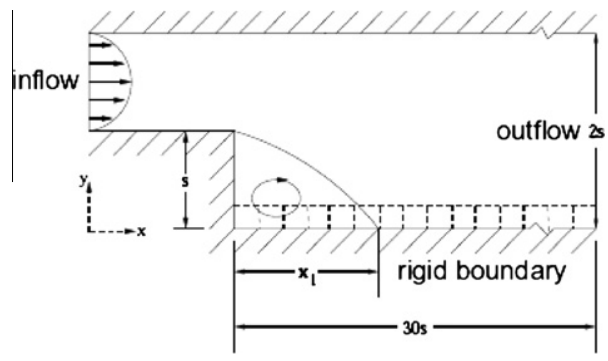

Fig. 10. Geometry of the backward facing step problem, showing a set of computational cells adjacent to the wall.

$u(x, 0)= \begin{cases}0.0, & \text { if } x \leqslant-1, \\ 0.5, & \text { if }-1<x<0 \\ 0.0, & \text { if } x \geqslant 0\end{cases}$

The exact solution is the rarefaction wave given by Ahmed (see [3]): $u(x, t)= \begin{cases}0.0, & \text { if } x \leqslant-1 \\ \frac{x+1}{t}, & \text { if }-1<x \leqslant \frac{t}{2}-1 \\ 0.5, & \text { if } \frac{t}{2}-1<x<\frac{t}{4}, \\ 0.0, & \text { if } x \geqslant \frac{t}{4}\end{cases}$

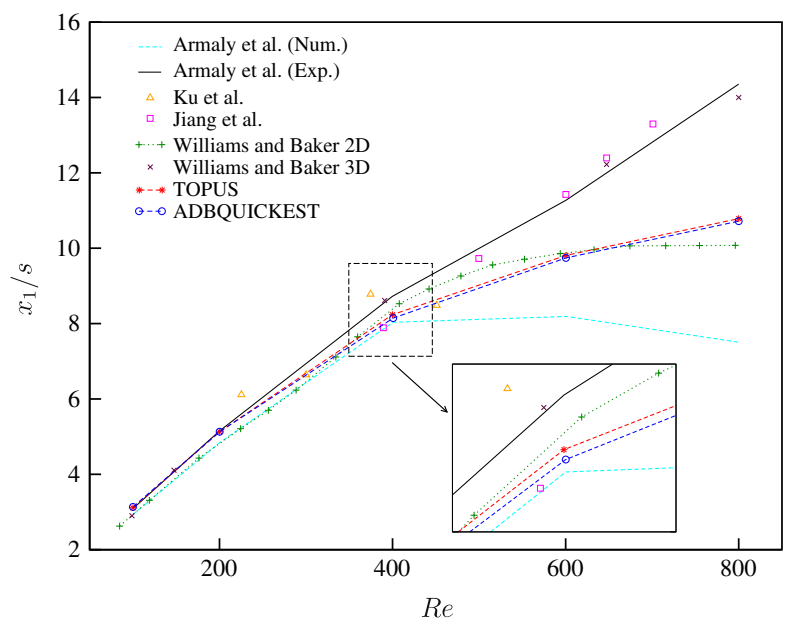

Fig. 11. Comparison between experimental data and numerical results for the size of the recirculation region length $x_{1}$ as a function of the Reynolds number. A closeup of the results are provided in the inset.

This problem consists of a jump from zero to one at $x=-1 / 3$ which creates an expansion fan, while the jump from one to zero at $x=1 / 3$ produces a shock wave. The purpose of this test is to check whether 


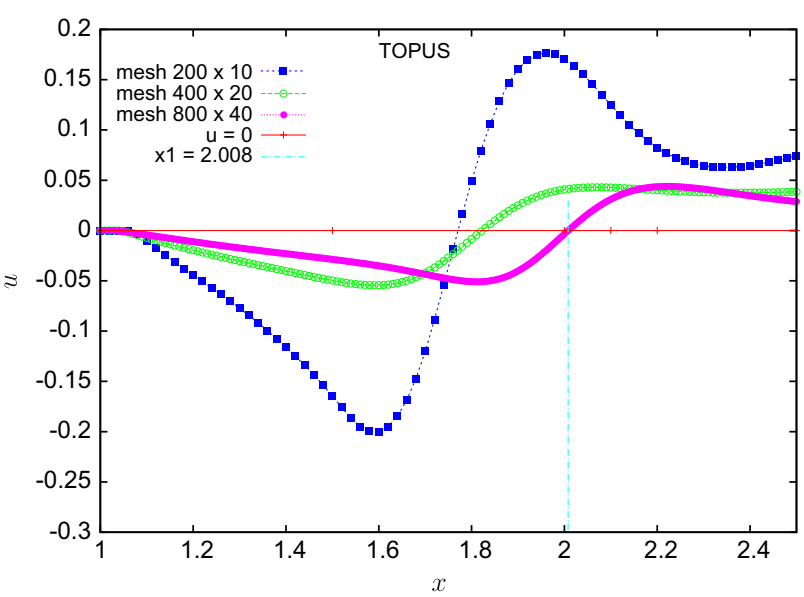

Fig. 12. Convergence test for the numerical solution for $u$ velocity of the flow over a backward facing step at Reynolds number $R e=400$.

the TOPUS scheme needs an additional smooth transition function (or not) to avoid entropy violation. A mesh size of $N=200 \mathrm{compu-}$ tational cells, final time $t=2, x \in[-1.5,1]$ and $\delta_{t}=0.01125$ were used in the simulation. The numerical results obtained with ADBQUICKEST, SMART, TOPUS, and VONOS schemes and the exact solution are presented in Fig. 6. Once again, it is seen that in comparison with the other methods TOPUS gives satisfactory results, capturing quite well the expansion fan and the shock wave without the need for adding an entropy correction formula.

Before concluding this section, we address the issue of nonlinear stability for the TOPUS scheme by checking the numerical time dependent total variation (TV) on progressively refined mesh sizes using the nonlinear problem (11) subject to the initial condition (29). Fig. 7 shows the TV calculated for $N=25,50,100$ and 200 computational cells. It can be seen that as time progresses the TV decreases or remains constant indicating that there is no loss of TV at local extrema.

Finally, the viscous Burgers' equation is solved in order to show that, for smooth solutions, the accuracy of the TOPUS scheme can be improved by implementating it in the flux function upwind reconstruction mode (similar to that appearing in Section 6, Eq. (84), in Ref. [74]). The boundary conditions are chosen equal to be $u(0, t)=\tanh (\operatorname{Re} / 4)$ and $u(1, t)=-u(0, t)$, with $R e=1 / v=20$. The initial condition is taken from the exact steady state solution in [22]. The observed order of accuracy with the TOPUS scheme, computed with both simplified and flux function reconstruction implementation modes, is depicted in Table 4. One can clearly see that, with the use of the flux function implementation, the TOPUS's convergence rate is improved. Also shown in Fig. 8 is the convergence history obtained with the TOPUS scheme, on a mesh size of 200 computational cells, using both simplified and flux function implementations. The explicit Euler method was used for the time March in this test case. The TOPUS scheme converges rapidly, at about thirteen orders of magnitude reduction in the residual. Therefore, in this paper, for all nonlinear problems involving convection-diffusion effects, we will use, for simplicity, the simplified implementation version of the TOPUS scheme given by Eqs. (12) and (13).

\subsection{D scalar convection-diffusion equation}

Having solved linear and nonlinear equations with different initial data, we now consider the most popular 1D scalar convectiondiffusion model (11) with $0<x<1$ and $\varepsilon=1$ (the so-called $1 \mathrm{D}$ boundary layer problem). The initial and boundary conditions are $u(x, 0)=0$ and $u(0, t)=0 ; u(1, t)=1, t \geqslant 0$, respectively. The exact steady state solution of this problem on the $i$ cell $\left((i \delta x)_{(0 \leqslant i \leqslant N)}\right)$ is given by (see [22] $) u_{i}=\left(1-\exp \left(i R e_{\delta}\right)\right) /(1-\exp (R e))$, where $R e_{\delta}=R e$ $\delta_{x}$ denotes the cell Reynolds number. The solution is obtained on a series of refined grids (from $N=80$ up to $N=640$ computational cells). A numerical convergence study is performed from calculations on several grids. Table 5 depicts the computed convergence rate when the ADBQUICKEST, SMART, Superbee, van Albada and TOPUS schemes are used for this problem for $R e=100$. It can be seen from this table that the $L_{1}, L_{2}$ and $L_{\infty}$ errors for the TOPUS scheme decrease with increasing grid points (mesh refinement), indicating convergence. In addition, the TOPUS scheme shows
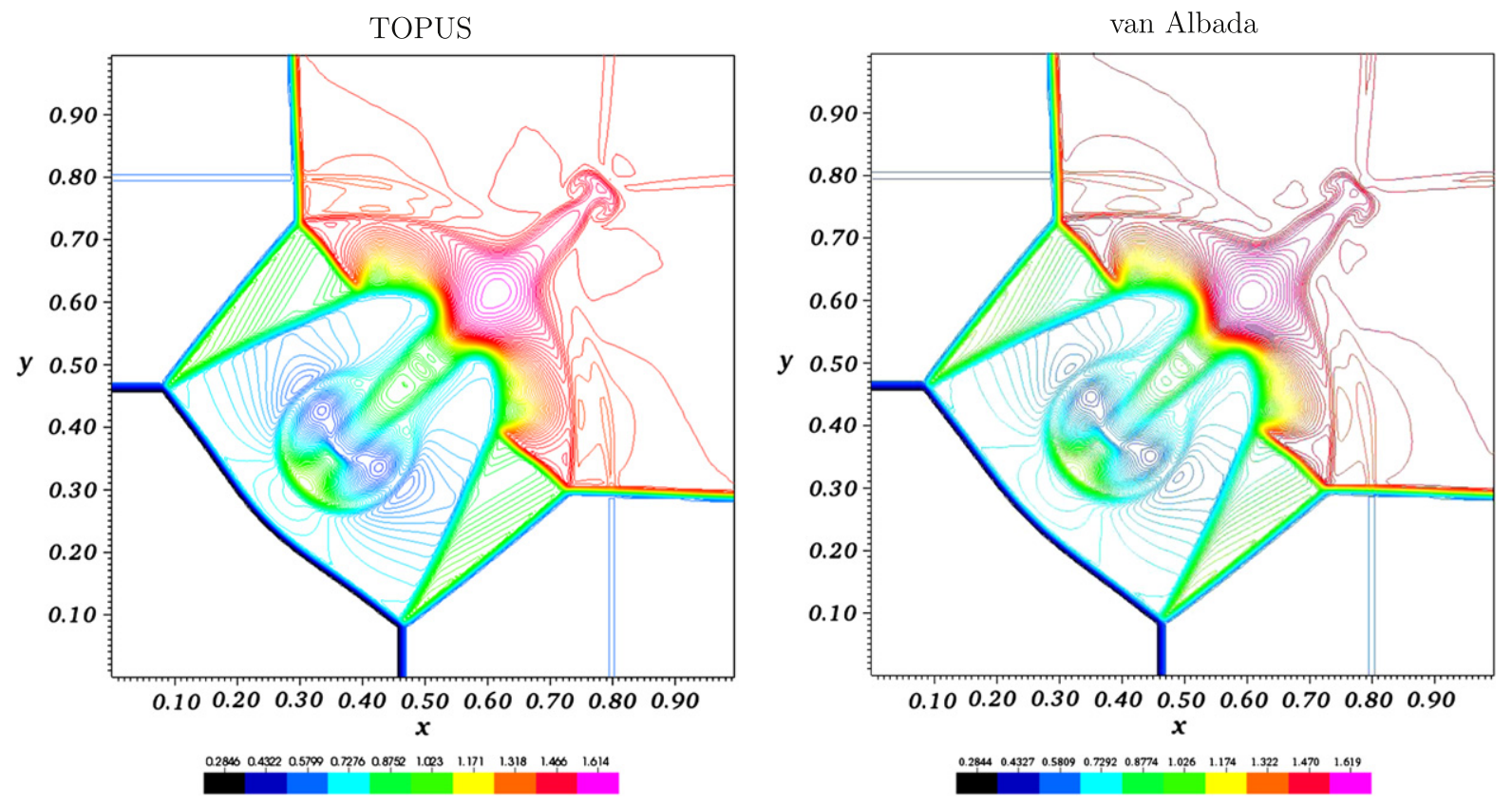

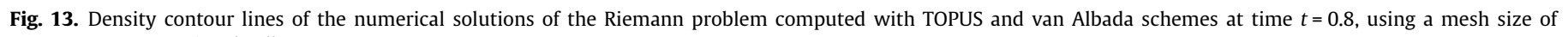
$200 \times 200$ computational cells. 

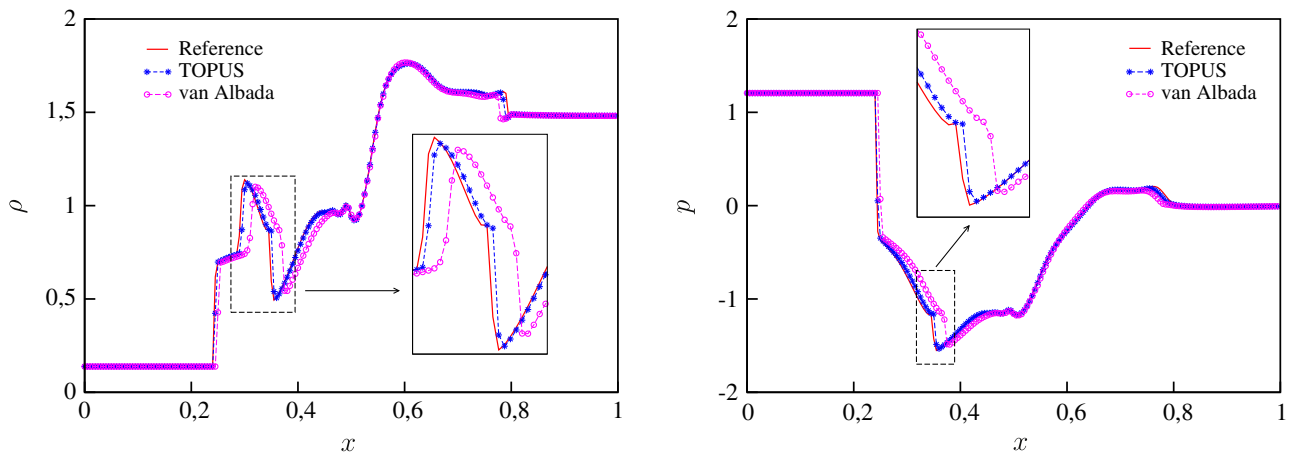

Fig. 14. Results for complex interacting flow structure problem along the diagonal $(x=y)$ line. On the left, density distribution and on the right pressure distribution.

improved numerical orders of accuracy compared with the global accuracy of the other schemes. The accuracy analysis performed here seems to indicate the dominant error is, in fact, the secondorder truncation error arising from the discretization of the diffusive term. In particular, the numerical diffusion introduced by TOPUS is significantly smaller than the physical diffusion coefficient $v$.

\subsection{D Shu-Osher's shock tube problem}

In order to verify that the TOPUS scheme is effective in preventing oscillations in unsteady flows, the 1D inviscid Euler Eq. (14) of gas dynamics was solved. The problem that was chosen is the Shu-Osher's shock tube [56] (see also [74]), that describes a shock interacting with smooth density fluctuations. This case provides a good test for examining the performance of the high order upwind schemes, because it possesses both strong discontinuous and smooth structures. Here, Eq. (14) is considered in the interval $[-1,3]$ with the initial condition

$$
(\rho, v, p)^{T}= \begin{cases}(3.86,2.63,10.33)^{T}, & \text { if } x \in[-1,0.8) \\ (1+0.2 \sin (5 x), 0,1)^{T}, & \text { if } x \in[0.8,3]\end{cases}
$$

In this case, two meshes $\left(N=200\right.$ with $\delta_{x}=0.02$ and $N=300$ with $\left.\delta_{x}=0.0133\right)$, time spacing $\delta_{t}=0.6 \delta_{x}$ and final time $t=1.0$ are used. The numerical results for density using ADBQUICKEST, LaxWendroff, Minmod, Superbee, van Leer and TOPUS schemes, and the reference solution (FOU scheme with $N=2000$ ) are presented in Fig. 9. One can observe that TOPUS provides reasonable resolution.

\section{6. $2 D$ incompressible flow over a backward facing step}

The flow over a backward facing step, comprehensively studied over the years and extensively used to analyze the quality of schemes, is computed here for laminar flow. The relevant conservation laws for 2D time-dependent incompressible fluid flow are the continuity (25) and the momentum (Navier-Stokes) (26) equations. The geometry of the problem is illustrated in Fig. 10. This problem is challenging computationally as it involves flow separation and recirculation. The size and location of the separation zone is very sensitive to the pressure gradient, thereby providing a good flow validation test case. Furthermore, there is extensive numerical and experimental data available in the literature. With a fully developed Poiseuille parabolic velocity profile prescribed at the

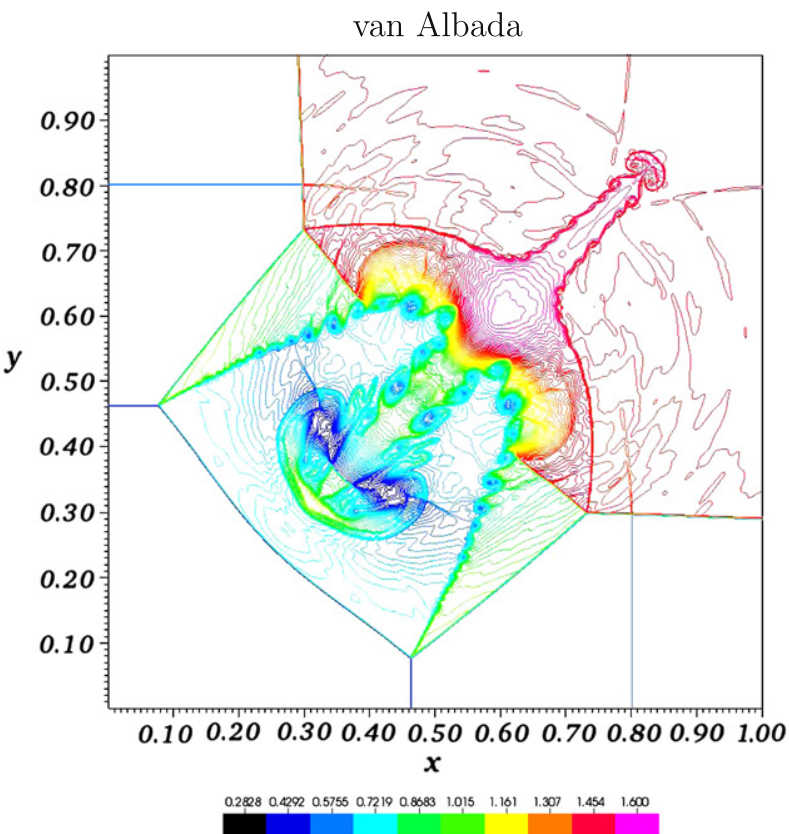

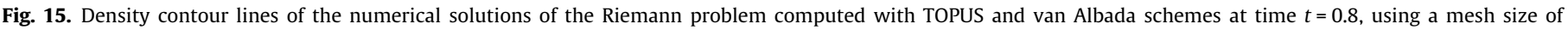
$1500 \times 1500$ computational cells. 


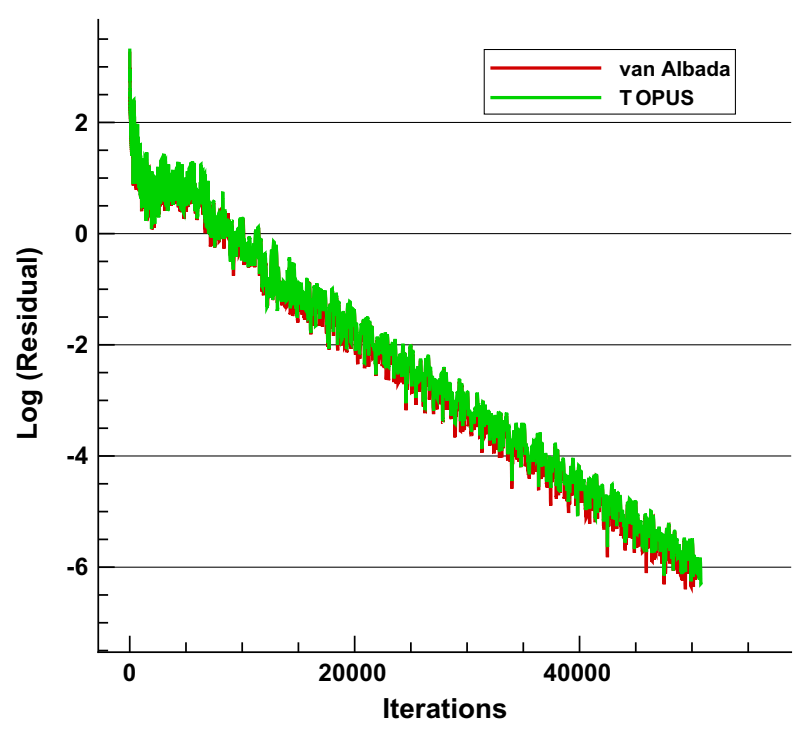

Fig. 16. Convergence histories with van Albada and TOPUS schemes for a NACA 0012 airfoil at $M_{\infty}=0.85$ and $\alpha=1^{\circ}$.

inlet section, we simulated numerically this fluid flow problem for a wide range of Reynolds numbers. These were based on the maximum velocity $U_{0}=U_{\max }=1 \mathrm{~m} / \mathrm{s}$ at the entrance section and the height of the step $s(s=D=0.1 \mathrm{~m})$. The dimension of the computational domain is $4.0 \mathrm{~m} \times 0.2 \mathrm{~m}$ and the total time of simulation is 100 seconds. A mesh size of $800 \times 40$ computational cells has been used in the simulations.

Fig. 11 graphically displays the evolution of reattachment lengths $x_{1}$, normalized by the step height $s$. With Reynolds numbers from 100 up to 800 , we have the following data: 2D numerical results of Armaly et al. [6] and Willians and Baker [72]; 3D numerical results of Willians and Baker [72], Ku et al. [36], Jiang et al. [34] and the experimental data of Armaly et al. [6]. We also have calculations using TOPUS and ADBQUICKEST schemes (no significant improvement was observed in the results obtained using the other schemes). The numerical results using ADBQUICKEST, for $0<R e<400$, show good agreement with the 2D results of Willians and Baker [72]; they would appear, however, to diverge from the data of Armaly et al. [6] and the 3D calculations. For $R e \geqslant 400$, the numerical results of both TOPUS and ADBQUICKEST give poor agreement with 3D data; this may be explained by 3D effects and, possibly, the turbulence transition in this high Reynolds number problem, as postulated by Ghia et al. [26]. This figure also shows a close-up, where it can be seen that the results obtained with the TOPUS scheme are marginally better than those obtained using the ADBQUICKEST scheme.

In addition, a convergence test of the numerical solution for (streamwise) velocity component $u$ was performed with a Reynolds number of 400 on three uniform meshes consisting of $200 \times 10,400 \times 20$ and $800 \times 40$ cells. This is illustrated in Fig. 12, which shows how the reattachment length $x_{1}$ was estimated (i.e. the change in the sign of the $u$ velocity profile adjacent to the lower bounding wall (see Fig. 10)).

\section{7. $2 D$ compressible Euler equations}

In this section, the TOPUS scheme is used to solve the $2 \mathrm{D}$ compressible Euler equations in conservative form (20) for steady and unsteady flows. The specific problems considered here are: (i) the shock-shock interaction problem originally defined by SchulzRinne et al. [53] (see also [15]) in the square domain

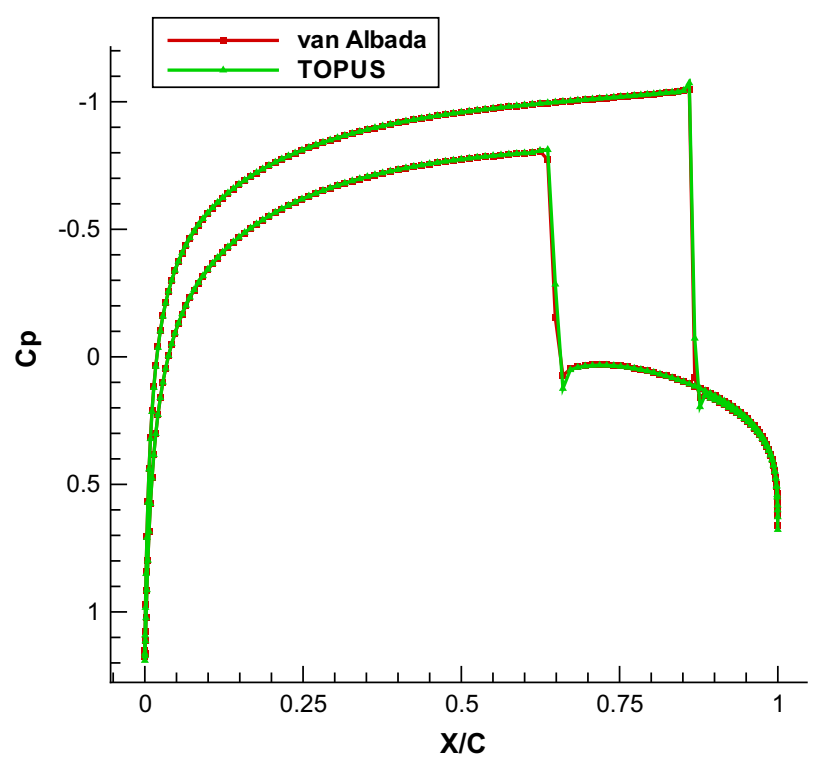

(a) Overall $C_{p}$ distributions

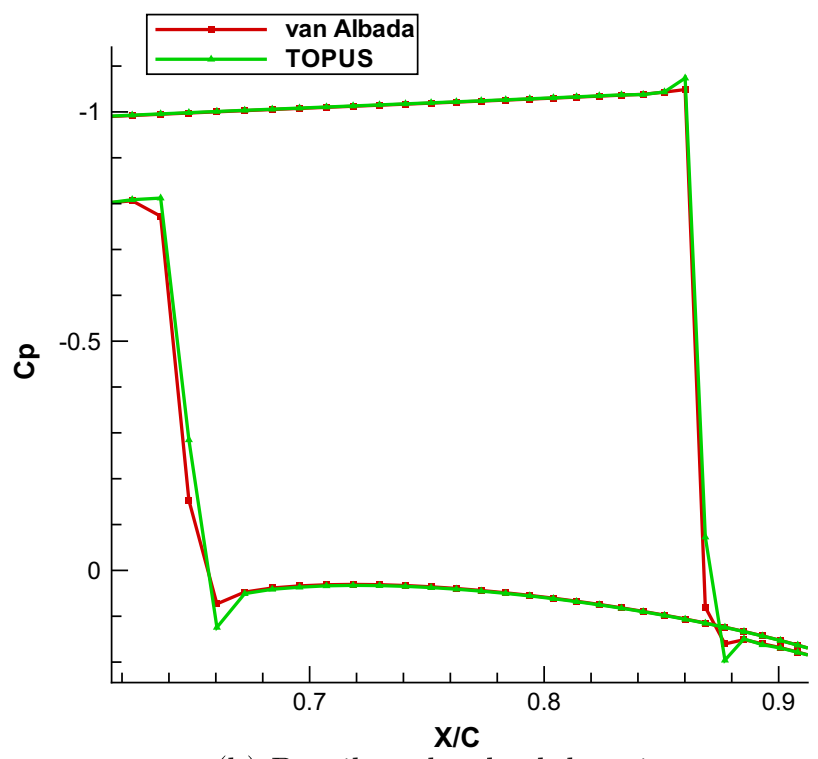

(b) Detail at the shock locations

Fig. 17. Comparison between numerical results obtained with TOPUS and van Albada limiters for the pressure coefficient distributions for a NACA 0012 airfoil at $M_{\infty}=0.85$ and $\alpha=1^{\circ}$.

$[0,1] \times[0,1]$; and (ii) the steady transonic flow around the NACA 0012 airfoil with $M_{\infty}=0.85$ and $\alpha=1^{\circ}$.

Computations for the shock-shock interaction problem were performed by using the CLAWPACK software [42], implemented with TOPUS, van Albada and MC limiter of van Leer [67] (see also [42] p. 115, or [27]). In the version of the CLAWPACK code that we have used, the Godunov's first-order explicit time marching method with second-order spatial corrections is implemented, where the flux functions are calculated by solving local Riemann problems; this allows the easy introduction of limiter functions to give high-resolution results. The solution of the problem using the MC limiter, on a mesh size of $2000 \times 2000$ computational cells, was selected as a reference solution, since this limiter has been one of the more widely used in engineering applications (see, for instance $[18,38]$ or $[27])$. This problem, a useful test to measure the smallness of the inherent numerical viscosity of the scheme, 


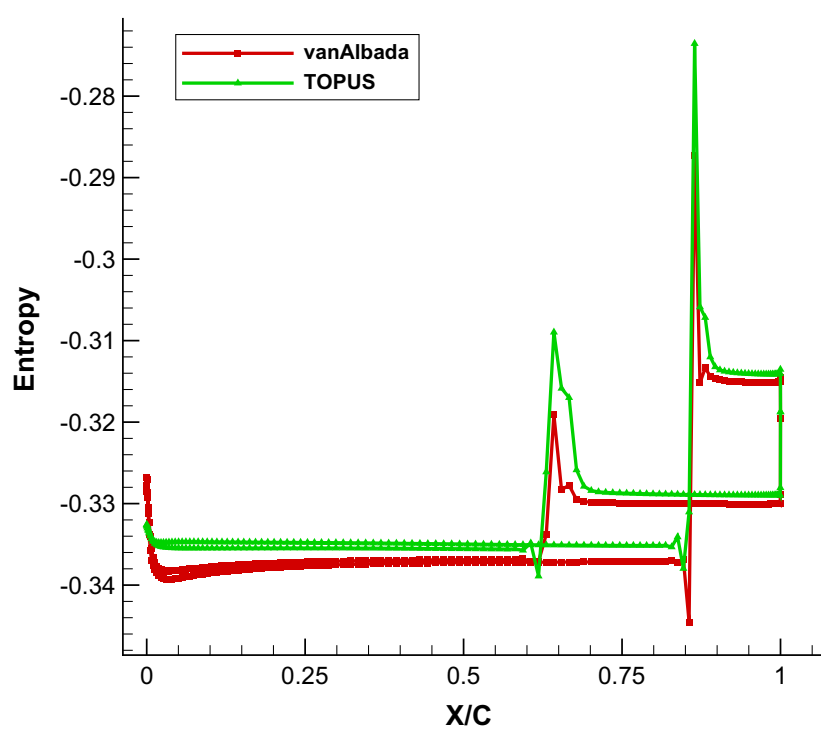

Fig. 18. Comparison of entropy generated at the airfoil surface by TOPUS and van Albada limiter calculations of the flow over a NACA 0012 airfoil at $M_{\infty}=0.85$ and $\alpha=1^{\circ}$.

arises frequently as a model for simulating thin shear layers or sharp interfaces between inviscid fluids [52]. The solutions were marched in time until time $t=0.8$ by using a mesh size of $200 \times 200$ computational cells and at CFL number of 0.8. Fig. 13 shows density contour lines computed with TOPUS and van Albada limiters. One can observe that TOPUS provides qualitatively the same resolution as van Albada except at the top right region, where the TOPUS limiter captures the vortical structures a little better. However, when the density and pressure profiles are plotted along the diagonal ( $x=y$ line), as shown in Fig. 14, significant differences between the TOPUS and the van Albada solutions can clearly be observed, indicating that TOPUS has behaved somewhat better than the van Albada limiter. In addition, to show that the TOPUS scheme is capable of capturing the complex interacting structures in the flow (i.e., vortex sheets), we repeat the numerical experiment shown in Fig. 13 using a mesh size of $1500 \times 1500$ computational cells. The density contour lines are depicted in Fig. 15, from which it can be seen that the TOPUS scheme provides a substantial improvement at the contact surface, where instabilities manifest themselves. So, it would appear that the TOPUS limiter introduces less numerical viscosity than van Albada.

We now focus on the specific AGARD test case (see [2]) of the steady inviscid compressible flow over a NACA 0012 airfoil at freestream Mach number $M_{\infty}=0.85$ and angle-of-attack $\alpha=1 \mathrm{deg}$. The objective of this test is to investigate whether the TOPUS scheme could resolve flows possessing strong shocks as well as the van Albada limiter, a widely used upwinding scheme for compressible flow computations. This classical case is computed using a mesh size of 251 points over the airfoil surface, 151 points in radial direction and the farfield boundary is set at 70 chords of radius. The solution is obtained using single-precision operations. The CFL number is set as a constant value of 0.7 and the maximum density residual for accepting convergence is chosen to be $10^{-7}$. Time March to steady state uses the 5-stage, 2 th-order accurate, explicit Runge-Kutta method presented in Ref. [32]. In Fig. 16, the convergence curves obtained with the van Albada and TOPUS schemes are presented, showing that both schemes converge, at about the same rate, with eight orders of magnitude reduction in the residual.

The pressure coefficient distributions, $C_{p}$, on the upper and lower surfaces of the airfoil obtained with TOPUS and van Albada limiters are plotted in Fig. 17. The overall views of the $C_{p}$ distri- butions are shown in Fig. 17a and detailed views of the upper and lower surface shock waves are shown in Fig. 17b. From these figures, it is seen that both TOPUS and van Albada limiters provide similar results, showing that the strength of the shock is in good agreement with the ones given in [2]. The results are also indicating that the shocks can be captured by the TOPUS scheme with 1-2 mesh points, whereas 3-4 points in the shock transition are observed when the van Albada limiter is used. The data in Fig. $17 \mathrm{~b}$ also indicate that TOPUS is slightly less dissipative than the van Albada limiter at the shock. Away from the shock waves, both TOPUS and van Albada schemes produce almost identical results.

Further investigation of these results can be achieved by inspecting the entropy generated by the numerical solutions. Hence, Fig. 18 presents the entropy generated at the airfoil surface by the two schemes for the same flight condition. Moreover, the entropy fields are shown in Fig. 19a and b for van Albada and TOPUS schemes, respectively. The clear conclusion from these figures is that the entropy generated by the two schemes is quite comparable. In Fig. 18, one can see that TOPUS creates slightly more entropy at the airfoil surface than the van Albada limiter. Again, these results emphasize that TOPUS has essentially the same shock capturing characteristics as the widely used van Albada limiter for such inviscid transonic applications.

Finally, drag and lift coefficients $\left(C_{d}\right.$ and $\left.C_{l}\right)$ are summarized in Table 6 . In this particular case, besides the comparison between TOPUS and van Albada schemes, we have included results for the present test case obtained by Amaladas and Kamath [1], Jameson and Martinelli [33] and by Pulliam and Barton [50]. The table also includes the range of values for lift and drag coefficients reported in [2]. Such data provide for a more quantitative comparison of the presently proposed scheme. One can see in Table 6 that the present results for lift and drag coefficients are between those provided by the van Albada limiter and those provided by the centered schemes. Again, the current results are very close to those provided by the van Albada limiter, except that we obtain a slightly higher value of lift coefficient, which is probably a consequence of the less dissipative behavior at the shock, as previously discussed, and also a somewhat higher drag coefficient. We believe that the higher drag coefficient is associated with the fact that TOPUS is generating slightly more entropy at the airfoil surface than the van Albada limiter, as indicated in Fig. 18. Hence, TOPUS produces more spurious drag than the van Albada limiter, explaining the higher $C_{d}$ values. However, one should notice that, clearly, such additional spurious drag is quite lower than what is generated by the other schemes compared in Table 6. Furthermore, the current results for both lift and drag coefficients are well within the ranges reported in [2].

\section{Closing remarks}

A high degree polynomial upwind-based finite difference scheme (TOPUS) has been introduced for the numerical solution of convection-dominated transport problems. This new scheme was derived from the application of the TVD/CBC stability criteria combined with the four conditions of Leonard [41]. TOPUS is presented in both the normalized variables of Leonard and also as a flux limiting technique, and has been shown to possess three important features: simplicity, robustness and generality of application. By setting the free parameter $\alpha$ equal to 2 , the scheme is then guaranteed to be oscillation-free; and, with this value of $\alpha$, the performance of the scheme was evaluated by solving a variety of test problems. These included the 1D/2D advection of scalars, the 1D Riemann problem for Euler's/Burgers' equation, the 2D Riemann problem for Euler's equation, the 2D incompressible Navier- 


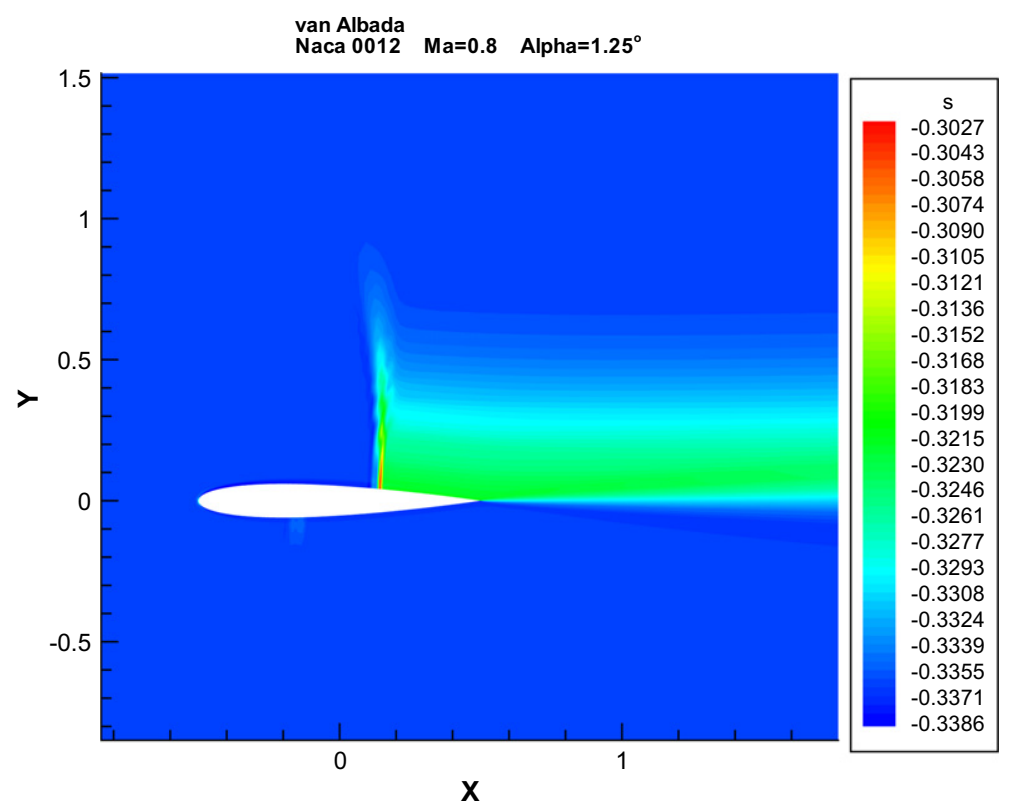

(a) van Albada limiter

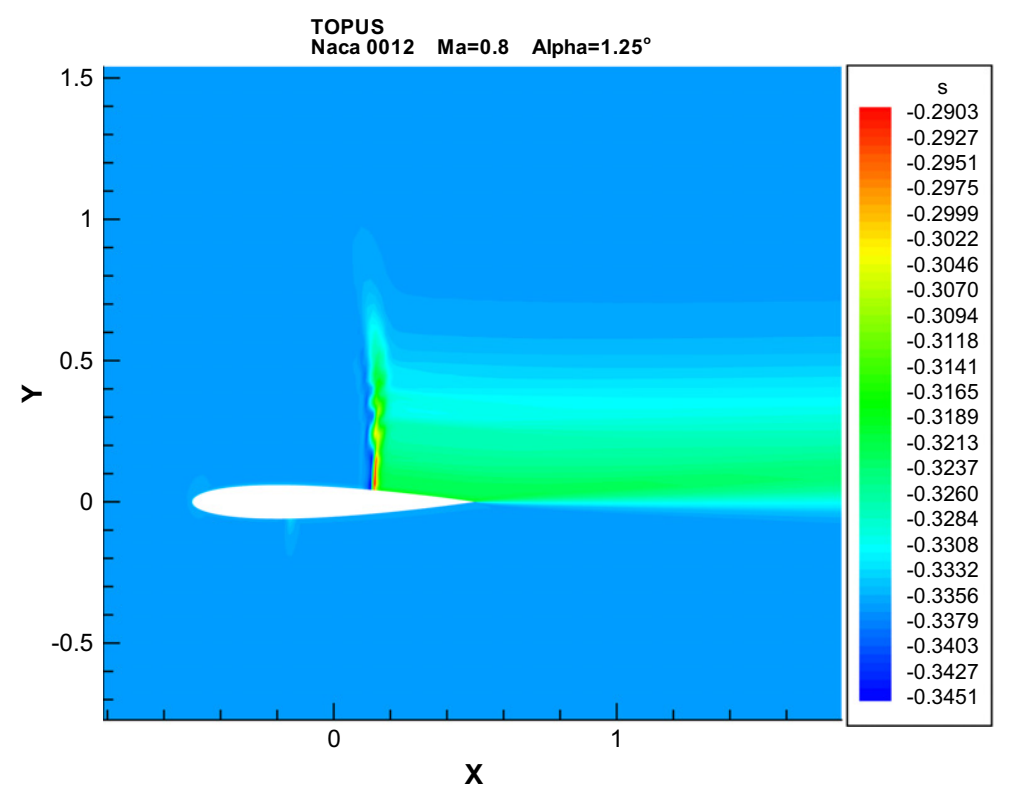

(b) TOPUS limiter

Fig. 19. Comparison of entropy fields for NACA 0012 airfoil at $M_{\infty}=0.85$ and $\alpha=1^{\circ}$.

Stokes equations and flow over a 2D airfoil. Reasonable results were obtained for all tests.

This study establishes the potential of the TOPUS scheme for solving a large class of complex problems and allows us to make the following points regarding TOPUS.

The TOPUS scheme can reach third-order accuracy in the case of linear advection, is second-order accurate in smooth regions of nonlinear problems, and is free from spurious oscillations around discontinuities. A comparison of the unit cost with other upwind schemes shows it in a favorable light.

For moderate CFL numbers and problems involving convection and diffusion, the parameter $\alpha$ should be chosen from [-2,2]; however, for problems involving shocks it is recommended that the user chooses $\alpha=2$. In particular, the choice of a good parameter $\alpha$ for simulations with the TOPUS scheme (especially for incom-
Table 6

Drag and lift aerodynamic coefficients for NACA 0012 airfoil at Mach 0.85 and $1^{\circ}$ angle of attack.

\begin{tabular}{lll}
\hline Scheme & $C_{d}$ & $C_{l}$ \\
\hline Amaladas and Kamath [1] & 0.0546 & 0.3799 \\
Jameson and Martinelli [33] & 0.0582 & 0.3861 \\
Pulliam and Barton [50] & 0.0604 & 0.3938 \\
van Albada & 0.0597 & 0.3617 \\
TOPUS & 0.0602 & 0.3616 \\
AGARD interval & $0.0464-0.0590$ & $0.330-0.3889$ \\
\hline
\end{tabular}

pressible flows over a backward facing step at high Reynolds numbers and compressible flows along NACA0012 airfoils with strong shocks) is important and always impacts upon the rate of convergence of steady-state solutions. 
The 1D numerical results in this paper show that TOPUS is a robust strategy for capturing shocks while maintaining a non-oscillatory profile, and when compared with reference solutions and the best existing schemes, TOPUS does as well and occasionally better.

However, although TOPUS competes well with existing schemes, such as SMARTER, 3rd WENO, MUSCL and ADBQUICKEST, it is certainly not always better. Its main advantage lies in the systematic nature of the scheme, its low cost and its fast implementation for prototyping and fluid model verification/validation.

In particular, TOPUS provides practically the same resolution as ADBQUICKEST without the need to tune the Courant parameter at each time step, and requires less computational time.

Overall, the TOPUS scheme is an alternative to the family of upwind schemes for simulating shock wave propagation and other phenomena where the nonlinear advection term requires special attention. We have numerically shown that the method can solve nonstationary as well as stationary problems in two space variables. In the transonic inviscid flow over NACA0012 airfoil computation, for example, TOPUS provided results compatible both with that given by van Albada and existing experimental data. However, as a perspective for improvement of steady compressible flow calculations, an extra effort will have to be undertaken in the future in order to couple the TOPUS scheme with an efficient implicit solver. For incompressible flows, TOPUS has proved to be an effective tool for resolving the delicate features and structures of a laminar flow over a backward facing step.

\section{Acknowledgments}

Financial support from the FAPESP under Grants 05/51458-0, 06/05910-1, 08/02673-4, 09/16954-8, 04/16064-9, and 09/158929 as well as CNPq under Grants 304201/2005-7, 312064/2006-3, 477858/2009-0 and 305447/2010-6 are gratefully appreciated. This work was also carried out in the framework of the Instituto Nacional de Ciência e Tecnologia em Medicina Assistida por Computação Científica (CNPq, Brazil).

\section{References}

[1] Amaladas JR, Kamath H. Accuracy assessment of upwind algorithms for steady-state computations. Comput Fluids 1998;93:941-62.

[2] AGARD Subcommittee C. Test cases for inviscid flow field methods, AGARD advisory report $211 ; 1985$. p. 6-27.

[3] Ahmed R. Numerical schemes applied to the burgers and Buckley-Leverett equations. MSc dissertation, University of Reading; 2004.

[4] Alves MA, Pinho FT, Oliveira. Effect of a high-resolution differencing scheme on finite-volume predictions of viscoelastic flows. J Non-Newton Fluid Mech 2000;93:287-314

[5] Alves MA, Oliveira PJ, Pinho FT. A convergent and universally bounded interpolation scheme for the treatment of advection. Int J Numer Methods Fluids 2003;41:47-75

[6] Armaly BF, Durst F, Pereira JCF, Schonung B. Experimental and theoretical investigation of backward facing step flow. J Fluid Mech 1983:127:473-96.

[7] Arora M, Roe PL. A well-behaved TVD limiter for high-resolution calculations of unsteady flow. J Comput Phys 1997;132:3-11

[8] Barth TJ, Jespersen DC. The design and application of upwind schemes on unstructured meshes. AIAA paper 89-0366. In: 27th AIAA aerospace sciences meeting, Reno, NV; 1989.

[9] Balsara DS, Shu CW. Monotonicity preserving weighted essentially nonoscillatory schemes with increasingly high order of accuracy. J Comput Phys 2000;160:405-52.

[10] Berthon C. Stability of the MUSCL schemes for the Euler equations. Commun Math Sci 2005;3:133-57.

[11] Bigarella EDV. Advanced turbulence modelling for complex aerospace applications. PhD thesis, Sao Jose dos Campos; 2007

[12] Bigarella EDV, Azevedo JLF. Advanced eddy-viscosity and Reynolds-stress turbulence model simulations of aerospace applications. AIAA paper no. 20062826. In: 24th AIAA applied aerodynamics conference, San Francisco, CA, USA, vol. 1 ; 2006. p. 1-39.

[13] Brandt A, Yavneh I. Inadequacy of first-order upwind difference schemes for some recirculating flows. J Comput Phys 1991;93:128-43.

[14] Burgers JM. A mathematical model illustrating the theory of turbulence. Adv Appl Mech 1948;1:171-99.
[15] Cada M, Torrilhon M. Compact third-order limiter functions for finite volume methods. J Comput Phys 2009;228:4118-45.

[16] Castelo A, Tomé MF, Cesar CNL, McKee S, Cuminato JA. Freeflow: an integrated simulation system for three-dimensional free surface flows. J Comput Visual Sci 2000;2:1-12.

[17] Chakravarthy SR, Osher S. High resolution applications of the Osher upwind scheme for the Euler equations, AIAA paper 83-1943. In: 6th Computational fluid dynamics conference; 1983. p. 363-73.

[18] Chassignet EP, Hurlburt HE, Smedstad OM, Halliwell GR, Hogan PJ, Wallcraft AJ, et al. The HYCOM (HYbrid Coordinate Ocean Model) data assimilative system. J Marine Syst 2007;65:60-83.

[19] Choi SK, Nam HY, Cho M. A comparison of higher-order bounded convection schemes. Comput Methods Appl Mech Eng 1995;121:281-301.

[20] Chorin AJ. Numerical solution of the Navier-Stokes equations. Math Comput 1968;22:745-62.

[21] Courant R, Isaacson E, Rees M. On the solution of nonlinear hyperbolic differential equations by finite differences. Commun Pure Appl Math 1952;5:243-55.

[22] Corre C, Lerat A. High-order residual-based compact schemes for advectiondiffusion problems. Comput Fluids 2008;37:505-19.

[23] Ferreira VG, Kurokawa FA, Queiroz RAB, Kaibara MK, Oishi CM, Cuminato JA, et al. Assessment of a high-order finite difference upwind scheme for the simulation of convection-diffusion problems. Int J Numer Methods Fluids 2009;60:1-26.

[24] Gaskell PH, Lau AKC. Curvature-compensated convective transport: SMART, a new boundedness-preserving transport algorithm. Int J Numer Methods Fluids 1988;8:617-41.

[25] Gauzha VG, Vorozhtsov EV. Numerical solutions for partial differential equations. New York: CRC Press; 1996.

[26] Ghia KN, Osswald GA, Ghia U. Analysis of incompressible massively separated viscous flows using unsteady Navier-Stokes equations. Int J Numer Methods Fluids 1989;9:1025-50.

[27] Godoy WF, Desjardin PE. On the use of flux limiters in the discrete ordinates method for 3D radiation calculations in absorbing and scattering media. J Comput Phys 2010;229:3189-213.

[28] Goodman JB, LeVeque RJ. A geometric approach to high resolution TVD schemes. SIAM J Numer Anal 1988;25:268-84.

[29] Harten A. High resolution schemes for hyperbolic conservation laws. J Comput Phys 1983;49:357-93.

[30] Harlow FH, Welch JE. Numerical calculation of time-dependent viscous incompressible flow of fluid with free surface. Phys Fluids 1965;8: 2182-9.

[31] Hubbard ME, Garcia-Navarro P. Flux difference splitting and the balancing of source terms and flux gradients. J Comput Phys 2000;165:89-125.

[32] Jameson A, Mavriplis D. Finite volume solution of the two-dimensional Euler equations on a regular triangular mesh. AIAA J 1986;24:611-8.

[33] Jameson A, Martinelli L. Mesh refinement and modeling errors in flow simulation. AIAA J 1998;36:676-86.

[34] Jiang G-S, Shu C-W. Efficient implementation of weighted ENO scheme. J Comput Phys 1996;126:202-28.

[35] Koren B. Upwind discretization of the steady Navier-Stokes equations. Int J Numer Methods Fluids 1990;11:99-117.

[36] Ku HC, Hirsch RS, Taylor TD, Rosenberg AP. A pseudospectral matrix element method for solution of the dimensional incompressible flows and its parallel implementations. J Comput Phys 1989;83:260-91.

[37] Kuan KB, Lin CA. Adaptive QUICK-based scheme to approximate convective transport. AIAA J 2000;38:2233-7.

[38] Lee L. A class of high-resolution algorithms for incompressible flows. Comput Fluids 2010;39:1022-32.

[39] Leonard BP. A stable and accurate convective modelling procedure based on quadratic upstream interpolation. Comput Methods Appl Mech Eng 1990;19:59-98.

[40] Leonard BP. Universal limiter for transient interpolation modeling of the advective transport equations: the ULTIMATE conservative difference scheme, NASA Technical Memorandum 100916, ICOMP-88-11; 1988

[41] Leonard BP. Simple high-accuracy resolution program for convective modeling of discontinuities. Int J Numer Methods Fluids 1988;8:1291-318.

[42] LeVeque RJ. Finite volume methods for hyperbolic problems. New York: Cambridge University Press; 2002.

[43] Lin H, Chieng CC. Characteristic-based flux limiter of an essentially third-order flux-splitting method for hyperbolic conservation laws. Int J Numer Methods Fluids 1991;13:287-307.

[44] Lin C-H, Lin CA. Simple high-order bounded convection scheme to model discontinuities. AIAA J 1997;35:563-5.

[45] McKee S, Tomé MF, Ferreira VG, Cuminato JA, Castelo A, Sousa FS, et al. The MAC method. Comput Fluids 2008;37:907-30.

[46] Naterer GF. Constructing an entropy-stable upwind scheme for compressible fluid flow. AIAA J 1999;37:303-12.

[47] Osher S. Convergence of generalized MUSCL schemes. SIAM J Numer Anal 1985;22:947-61.

[48] Patankar SV. Numerical heat transfer and fluid flows. New York: Hemisphere Publishing Corporation; 1980.

[49] Piperno S, Depeyre S. Criteria for the design of limiters yielding efficient high resolution TVD schemes. Int J Numer Methods Fluids 1998;27:183-97.

[50] Pulliam TH, Barton JT. Euler computations of AGARD working 07 airfoil test cases. AIAA paper, 85-0018; 1985. 
[51] Roe P, Pike J. Efficient construction and utilization of approximate Riemann solutions. Comput Methods Appl Sci Eng 1984;6:499-518.

[52] Samtaney R, Pullin DI. On initial-value and self-similar solutions of the compressible Euler equations. Phys Fluids 1996;8:2650-5.

[53] Schulz-Rinne CW, Collins JP, Glaz HM. Numerical solution of the Riemann problem for two-dimensional gas dynamics. SIAM J Sci Comput 1993;14: 1394-414.

[54] Smith RM, Hutton AG. The numerical treatment of advection: a performance comparison of currents methods. Numer Heat Transfer 1982;5:439-61.

[55] Shin JK, Choi YD. Study on the improvement of the convective differencing scheme for the high-accuracy and stable resolution of the numerical solution. Trans KSME 1992;16:345-60.

[56] Shu C-W, Osher S. Efficient implementation of essentially non-oscillatory shock capturing schemes. J Comput Phys 1989;83:32-78.

[57] Song B, Liu GR, Lam KY, Amano RS. On a higher-order bounded discretization scheme. Int J Numer Methods Fluids 2000;32:881-97.

[58] Spalding DB. A novel finite difference formulation for differential expressions involving both first and second derivatives. Int J Numer Methods Eng 1972;4:551-9.

[59] Strikwerda JG. Upwind differencing, false scaling, and nonphysical solutions to the driven cavity problem. J Comput Phys 1982;47:303-7.

[60] Sweby PK. High resolution schemes using flux limiters for hyperbolic conservation laws. SIAM J NumerAnal 1984;21:995-1011.

[61] Tang H, Warnecke G. A Runge-Kutta discontinuous Galerkin method for the Euler equations. Comput Fluids 2005;34:375-98.

[62] Toro EF. Riemann solver and numerical methods for fluid dynamics. 2nd ed. Berlin (Heidelberg): Springer-Verlag; 1999.

[63] Tremback CJ, Powell J, Cotton WR, Pielke RA. The forward in-time upstream advection scheme: extension to higher orders. Mon Weather Rev 1987;115: 540-55.

[64] van Albada GD, van Leer B, Roberts WW. A comparative study of computational methods in cosmic gas dynamics. Astron Astrophys 1982;108:76-84.

[65] Varonos A, Bergeles G. Development and assessment of a variable-order nonoscillatory scheme for convection term discretization. Int J Numer Methods Fluids 1998;26:1-16
[66] van Leer B. Towards the ultimate conservative difference scheme II. Monotonicity and conservation combined in a second-order scheme. J Comput Phys 1974;14:361-70.

[67] van Leer B. Towards the ultimate conservative difference scheme IV. A new approach to numerical convection. J Comput Phys 1977;23:276-99.

[68] van Leer B. Towards the ultimate conservative difference scheme V. A secondorder sequel to Godunov's method. J Comput Phys 1979;32:101-36.

[69] van Leer B. Upwind and high-resolution methods for compressible flow: from donor cell to residual-distribution schemes. Commun Comput Phys 2006;1:192-206.

[70] Warming RF, Beam RM. Upwind second-order difference schemes and applications in aerodynamic flows. AIAA J 1976;14:1241-9.

[71] Waterson NP, Deconinck H. Design principles for bounded higher-order convection schemes - a unified approach. J Comput Phys 2007;224:182-207.

[72] Williams PT, Baker AJ. Numerical simulations of laminar flow over a 3D backward-facing step. Int J Numer Methods Fluids 1997;24:1159-83.

[73] Xue S-C, Phan-Thien N, Tanner RI. Upwinding with deferred correction (UPDC): an effective implementation of higher-order convection schemes for implicit finite volume methods. J Non-Newton Fluid Mech 2002;108:1-24.

[74] Yamaleev NK, Carpenter MH. Third-order energy stable WENO scheme. J Comput Phys 2009;228:3025-47.

[75] Zhang J, Jackson TL. A high-order incompressible flow solver with WENO. J Comput Phys 2009;228:2426-42.

[76] Zijlema M. On the construction of a third-order accurate monotone convection scheme with application to turbulent flows in general domains. Int J Numer Methods Fluids 1996;22:619-41.

[77] Zhou G, Davidson L, Olsson E. Transonic inviscid/turbulent airfoil flow simulations using a pressure based method with high order schemes. Lect Notes Phys 1995;453:372-7.

[78] Zhou T, Li Y, Shu C-W. Numerical comparison of WENO finite volume and Runge-Kutta discontinuos Galerkin methods. J Sci Comput 2001;16:145-71.

[79] Zhu J. On the higher-order bounded discretization schemes for finite volume computations of incompressible flows. Comput Methods Appl Mech Eng 1992;98:345-60. 